\title{
JOINT PRIORITY-BASED CONGESTION CONTROL IN MULTI-PATH AND MULTI-HOP WIRELESS SENSOR NETWORKS
}

\author{
by \\ Zhibin Li, B.Eng \\ A Thesis submitted to the Faculty of Graduate Studies \\ Carleton University \\ In partial fulfillment of the requirements for the degree of \\ Master of Applied Science \\ in Electrical Engineering \\ Ottawa-Carleton Institute for Electrical and Computer Engineering \\ Department of Systems and Computer Engineering \\ Faculty of Engineering \\ Carleton University \\ Ottawa, Ontario, Canada
}

(C) Zhibin Li, Ottawa, Canada, 2007 


$\begin{array}{ll}\begin{array}{l}\text { Library and } \\ \text { Archives Canada }\end{array} & \begin{array}{l}\text { Bibliothèque et } \\ \text { Archives Canada }\end{array} \\ \begin{array}{l}\text { Published Heritage } \\ \text { Branch }\end{array} & \begin{array}{l}\text { Direction du } \\ \text { Patrimoine de l'édition }\end{array} \\ \begin{array}{l}\text { 395 Wellington Street } \\ \text { Ottawa ON K1A 0N4 } \\ \text { Canada }\end{array} & \begin{array}{l}\text { 395, rue Wellington } \\ \text { Ottawa ON K1A ON4 } \\ \text { Canada }\end{array}\end{array}$

Your file Votre référence ISBN: 978-0-494-33657-1 Our file Notre référence ISBN: 978-0-494-33657-1

NOTICE:

The author has granted a nonexclusive license allowing Library and Archives Canada to reproduce, publish, archive, preserve, conserve, communicate to the public by telecommunication or on the Internet, loan, distribute and sell theses worldwide, for commercial or noncommercial purposes, in microform, paper, electronic and/or any other formats.

The author retains copyright ownership and moral rights in this thesis. Neither the thesis nor substantial extracts from it may be printed or otherwise reproduced without the author's permission.
AVIS:

L'auteur a accordé une licence non exclusive permettant à la Bibliothèque et Archives Canada de reproduire, publier, archiver, sauvegarder, conserver, transmettre au public par télécommunication ou par l'Internet, prêter, distribuer et vendre des thèses partout dans le monde, à des fins commerciales ou autres, sur support microforme, papier, électronique et/ou autres formats.

L'auteur conserve la propriété du droit d'auteur et des droits moraux qui protège cette thèse. $\mathrm{Ni}$ la thèse ni des extraits substantiels de celle-ci ne doivent être imprimés ou autrement reproduits sans son autorisation.
In compliance with the Canadian

Privacy Act some supporting forms may have been removed from this thesis.

While these forms may be included in the document page count, their removal does not represent any loss of content from the thesis.
Conformément à la loi canadienne sur la protection de la vie privée, quelques formulaires secondaires ont été enlevés de cette thèse.

Bien que ces formulaires aient inclus dans la pagination, il n'y aura aucun contenu manquant.

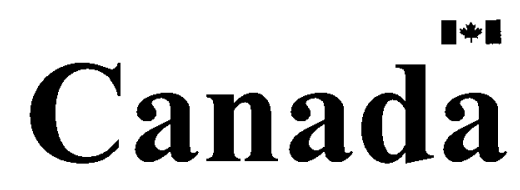




\begin{abstract}
In this thesis, we proposed a congestion control algorithm called Joint Priority Algorithm (JPA). In the JPA, each sensor node maintains a set of variables storing the values of Joint Priority $(J P)$ of its upstream neighbors. When congestion occurs in this node, it will allocate bandwidth proportional to these values. The sensor node calculates the $J P$ based on only local information instead of per-flow one, which makes it possible to apply this algorithm in multi-path routing WSNs.
\end{abstract}

The proposed algorithm allows packets from data sources to the base station to follow an arbitrary set of forwarding paths and allocates bandwidth fairly among different data sources when congestion occurs. Moreover, this algorithm guarantees end-to-end priority-based fairness, which makes bandwidth allocation biased toward important data sources.

Much of the work in this thesis has focused on achieving end-to-end fairness and end-to-end weighted fairness in congestion control protocols for multi-path routing WSNs. Energy efficiency performance will be the main target in our future work. 


\section{ACKNOWLEGEMENTS}

First and foremost, I would like to express my sincere appreciation and gratitude to my supervisor, Dr.Peter Liu, for providing valuable advice and guidance whenever I need, and for directing and supporting my work.

I also would like to thank my colleagues in the lab and my friends for sharing their wonderful idea with me.

Finally, I also would like to deeply thank my family for their support and encouragement through this effort, especially to my dear wife, Jane for her invaluabe and consistent support during the sometimes difficult periods that have led to the production of this thesis. 


\section{Contents}

1 INTRODUCTION 1

1.1 Motivation ....................... 3

1.1.1 Wireless sensor networks: An introduction . . . . . . . . . 3

1.1.2 Applications of wireless sensor networks . . . . . . . . 8

1.1.3 The need for congestion control in WSNs . . . . . . . . 10

1.2 Congestion Control for Wireless Sensor Networks . . . . . . . . . 11

1.2 .1 Congestion detection $\ldots \ldots \ldots \ldots \ldots$

1.2 .2 Congestion notification $\ldots \ldots \ldots \ldots \ldots \ldots$

1.2.3 Rate adjustment . . . . . . . . . . . . . . 13

1.3 Fairness Issues in Congestion Control . . . . . . . . . . . . . . . 14

1.4 Previous Research Work and Problem Statement _. . . . . . . . 15

1.5 Objectives of this Thesis $\ldots \ldots \ldots \ldots$

1.6 Thesis Contributions . . . . . . . . . . . . . . . . . . . . 19

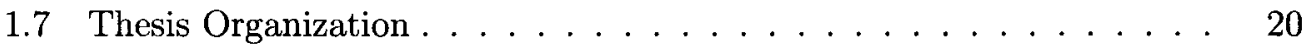

2 BACKGROUND $\quad 22$

2.1 Carrier Sensing Multiple Access (CSMA) . . . . . . . . . . . 22 
2.2 Rate Adjustment Algorithm . . . . . . . . . . . . . . . 26

2.2.1 Additive increase multiplicative decrease (AIMD) $\ldots \ldots 26$

2.2 .2 Token bucket algorithm . . . . . . . . . . . 29

2.3 Routing Protocol in WSNs . . . . . . . . . . . . . . . . 31

2.3.1 Challenges and design issues in WSNs routing protocol . . . 31

2.3.2 Classification of routing protocol in WSNs . . . . . . . . 34

2.4 Performance Metrics of Congestion Control Protocols in WSNs . . . . 42

2.4 .1 Energy efficiency . . . . . . . . . . . . . 43

2.4.2 End-to-end fairness and end-to-end weighted fairness . . . . 44

3 JOINT PRIORITY ALGORITHM $\quad 46$

3.1 Joint Priority Algorithm Notations . . . . . . . . . . . . . . 47

3.2 Network Model . . . . . . . . . . . . . . . . . . . . 50

3.3 Node Model . . . . . . . . . . . . . . . . . . . . . . . . 52

3.4 The Joint Priority Algorithm . . . . . . . . . . . . . . . 54

3.4.1 Challenges of end-to-end fairness and end-to-end weighted fairness 55

3.4 .2 The proposed algorithm . . . . . . . . . . 57

4 SIMULATION ENVIRONMENT AND RESULTS ANALYSIS 65

4.1 Simulation Environment . . . . . . . . . . . . . 66

4.2 Simulation Results Analysis . . . . . . . . . . . . . . . 67

4.2 .1 Fairness comparison . . . . . . . . . . 67

4.2.2 Achieving end-to-end weighted fairness . . . . . . . . 72

4.3 Summary of Simulation Results . . . . . . . . . . . . . . . 77 
$\begin{array}{lll}5 & \text { CONCLUSIONS } & \mathbf{7 8}\end{array}$

5.1 Conclusions . . . . . . . . . . . . . . . . . 78

5.2 Future Work . . . . . . . . . . . . . . . . . . 80

$\begin{array}{lc}\text { A Glossary of Terms } & 82\end{array}$

$\begin{array}{lr}\text { B Bibliography } & \mathbf{8 5}\end{array}$ 


\section{List of Tables}

4.1 Fairness simulation parameters $\ldots \ldots \ldots 67$

4.2 weighted fairness simulation parameters . . . . . . . . . 72 


\section{List of Figures}

1.1 Functional component of a typical wireless sensor node $[3] \ldots \ldots$

1.2 Mica2 sensor node $\ldots \ldots \ldots \ldots \ldots$

1.3 Clustered sensor networks with 12 nodes $\ldots \ldots \ldots 7$

1.4 Healthcare application of WSNs $\ldots \ldots \ldots \ldots$

1.5 Network performance curve . . . . . . . . . . . . . . . . 10

1.6 Tree-based network model in single-path routing WSNs . . . . . . 18

2.1 Hidden terminal problem . . . . . . . . . . . . . . . . . . 24

2.2 Exposed terminal problem . . . . . . . . . . . . . 25

2.3 RTS/CTS protocol . . . . . . . . . . . . . 26

2.4 TCP AIMD Mechanism $\ldots \ldots \ldots \ldots$

2.5 Rate-based AIMD Model . . . . . . . . . . . . . . . . . 29

2.6 Token bucket model[15] . . . . . . . . . . . . . . . . . . . 30

2.7 The classification of routing protocols in WSNs $\ldots \ldots \ldots 34$

2.8 Node-disjoint multipath routing . . . . . . . . . . . . . . . . . 39

2.9 Braided multipath routing . . . . . . . . . . . . . . 40

3.1 Network model in JPA . . . . . . . . . . . . . . . . . . . 47

3.2 Node model in JPA . . . . . . . . . . . . . . . . . 48

$\mathrm{ix}$ 
3.3 Weighted fair queue model . . . . . . . . . . . . . . 54

3.4 A topology of local fairness example . . . . . . . . . . . 56

3.5 Flowchart of token bucket algorithm . . . . . . . . . . . 64

4.1 Network simulation topology $\ldots \ldots \ldots 66$

4.2 Simulation result of end-to-end fairness in no congestion control . . 68

4.3 Simulation result of end-to-end fairness in CODA . . . . . . . . 69

4.4 Simulation result of end-to-end fairness in PCCP . . . . . . . 70

4.5 Simulation result of end-to-end fairness in JPA . . . . . . . . 71

4.6 Simulation result 1 of end-to-end weighted fairness in JPA $\ldots \ldots 73$

4.7 Simulation result 2 of end-to-end weighted fairness in JPA $\ldots . .74$

4.8 Simulation result 3 of end-to-end weighted fairness in JPA $\ldots \ldots 75$

4.9 Simulation result 4 of end-to-end weighted fairness in JPA $\ldots 76$ 


\section{Chapter 1}

\section{INTRODUCTION}

As small, low-cost sensors become technically and economically viable, wireless sensor networks (WSNs) will play an important role in sensing, collecting, propagating and processing information[1]. The sensor nodes of a WSN sense physical phenomena and transmit information of interest to base stations. End users can thus access and use the information obtained through base stations.

WSNs have a wide spectrum of applications, including environment monitoring, military operations, intelligent buildings, etc. WSNs can be classified in two categories based on their data collection and transmission: event-based and dataflow-based [2]. Event-based WSNs use sensor nodes to send data packets to base stations only when an event occurs. Data flow based WSNs report data packets to base stations periodically. Both types, however, produce convergent data flows from source nodes 
to base stations, which can potentially cause transmission congestion. For event-based WSNs, congestion results when many sensor nodes detect an event and send the data at the same time. For example, an environment monitoring WSN may consist of only one or a few base stations, but may have hundreds or thousands of tiny sensor nodes that spread across a geographical area. Because they send highly frequent transmissions, congestion is more likely to occur in dataflow-based WSNs than eventbased ones. In order to save the limited energy of sensor nodes and prolong the lifetime of the entire WSN, congestion must be controlled or avoided.

There are two types of congestion in WSNs. One is radio collision, which occurs when many sensors attempt to send data simultaneously. A classic solution to this problem is the exponential random back off method. Another type is buffer-based congestion, which can easily occur in a WSN when the packets converge toward a sink. Buffer management based mechanisms are proposed to avoid this kind of congestion.

Many congestion control protocols have been proposed for WSNs. The efficiency of a congestion control protocol depends mainly on the following two performance criteria:

- Energy efficiency

- End-to-end fairness and end-to-end weighted fairness

Energy efficiency is required to prolong the lifetime of WSNs, while end-to-end fairness 
and end-to-end weighted fairness guarantees that each node can achieve equal or weighted fair throughput when congestion occurs.

In this thesis, we develop a novel joint priority based congestion control protocol for WSNs. This protocol will eliminate congestion and achieve end-to-end priority based fairness in multi-path and multi-hop WSNs.

In this chapter, we will outline the motivation of this thesis and introduce congestion control algorithms in WSNs. Then we will review the previous work related to congestion control. Finally, the objectives and contributions of this thesis will be presented.

\subsection{Motivation}

\subsubsection{Wireless sensor networks: An introduction}

\section{Wireless Sensor Nodes}

A WSN may consist of hundreds or thousands of low cost sensor nodes which may have either a fixed location or been randomly deployed. A sensor node is a device that has the ability of sensing, signal processing and wireless communication. Figure 1.1 provides a schematic diagram of the functional components of a typical wireless sensor node. The transducer unit converts the sensed analog signals to digital signals. 
The control unit receives digital input from the sensing unit and performs protocol operations. The memory unit is used to save data. An Arithmetic and Logic Unit (ALU) is employed to calculate and compare the information collected. The timer unit schedules data transmission. The protocol unit is used to implement the network protocol functions, while the CPU controls all the above units. The radio unit transmits and receives the packets over the wireless link. The battery unit is the power source for the entire sensor node. Due to their small size, these units/components have a number of limitations, such as limited power and memory space. A number of design issues must be addressed to achieve effective and efficient operation of wireless sensor networks[3].

Due to the limited capabilities of the sensor node, a specially designed operation system is required. For example, Figure 1.2 depicts the Mica2 sensor node whose operation system is called TinyOS. This node was initially developed at the University of California, Berkeley. The programming language of TinyOS is stylized C, which uses a custom compiler called NesC. The Mica2 sensor node is one of the most popular research platforms today.

\section{Wireless Sensor Networks}

A wireless sensor network may consist of several base stations (sinks) and many sensor nodes. The main task of WSNs is to collect information. For example, to sense the 


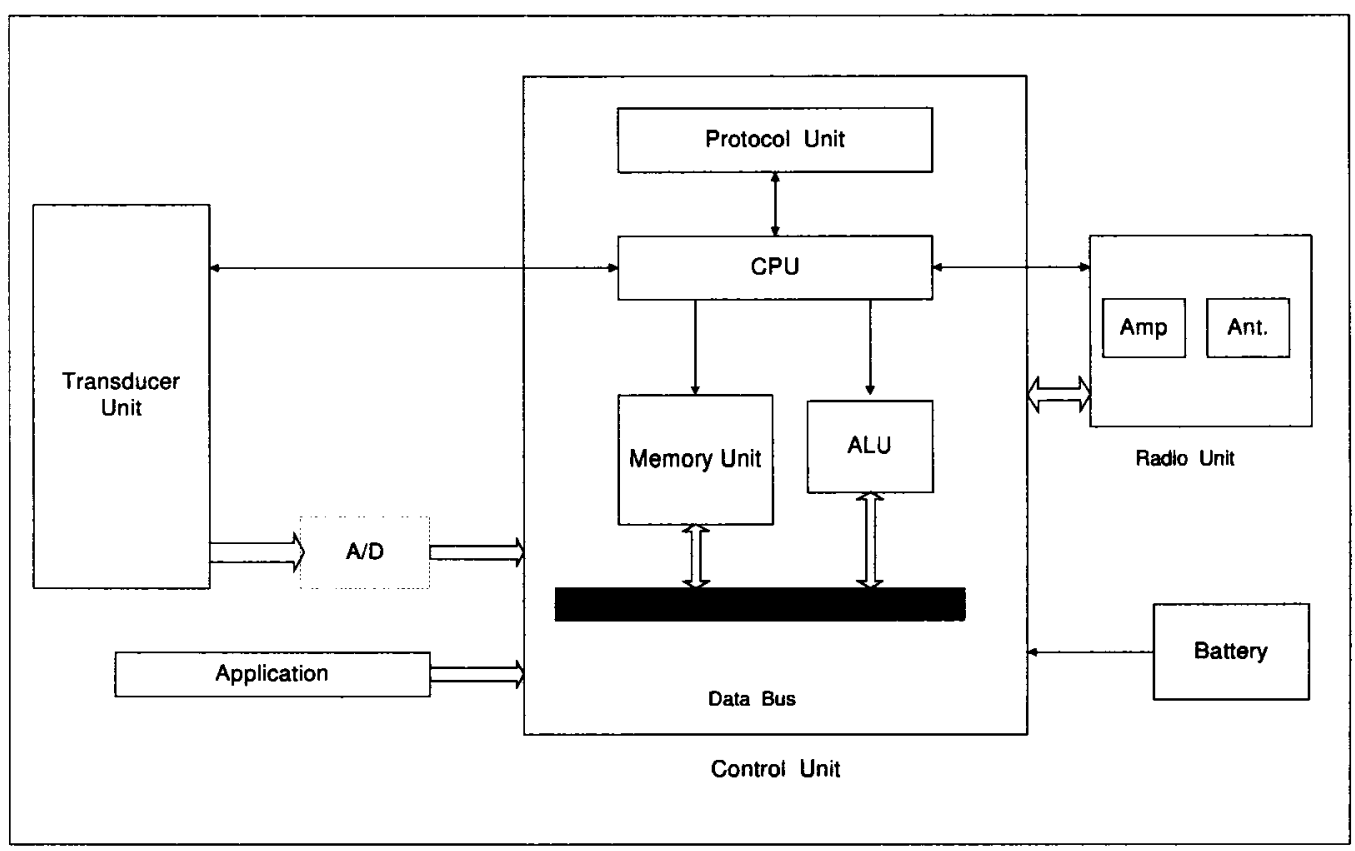

Figure 1.1: Functional component of a typical wireless sensor node [3]

environment for specific phenomena. The sensed data are then transmitted to the base station, for access by the user. The deployment area of WSNs in most cases is very large. However, due to their limited radio capabilities, sensor nodes have a relatively short transmission range. Therefore, the data must be relayed through intermediate nodes towards the sink. It may be advantageous to use a multi-hop path to the sink which consists of shorter links rather than to use a single long connection. This is because long range communication costs more energy than short range communication.

In many applications, the position of a sensor node cannot be predetermined. This 


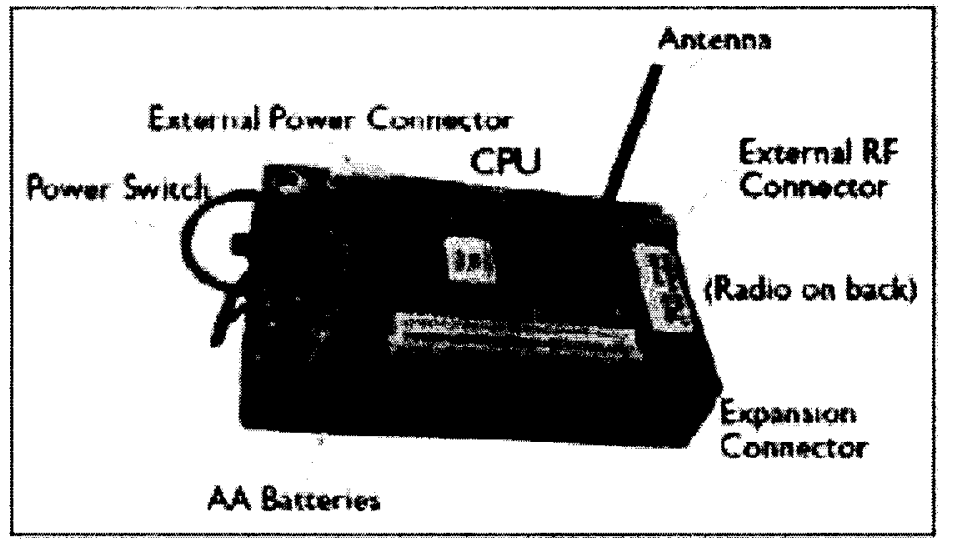

Figure 1.2: Mica2 sensor node

means random deployment in the inaccessible area. Therefore the WSN must possess self-organizing capabilities. For example, an aircraft can spread a large number of sensor nodes over the environment without having the prior knowledge of where each sensor is being individually placed.

Another unique feature of WSNs is their processing abilities. The sensor nodes carry out local computation and comparison instead of sending raw data to the base station. However, their limited power and size mean that the sensor nodes are able to perform only simple computation and comparison locally.

Because sensor nodes have limited power, computational capacities and memory, sensed data are not sent directly to the base station over the network. Instead, WSNs usually employ an aggregation approach. The aggregation summarizes current data received from neighboring nodes in some or all of a sensor network. Finally, the filtered data are forwarded to the next hop. The aggregation approach reduces the 
total amount of data exchanged between nodes. This approach also saves energy because radio communication is by far the most power intensive task, and this cost rises dramatically with distance. For example, the energy cost of transmitting $1 \mathrm{~Kb}$ data 100 meters in a free-space is approximately 3 Joules, while a general purpose processor with 100 MIPS/W power could efficiently execute 3 million instructions for the same amount of energy [4]. Another approach similar to the aggregation approach is clustering. Sensor nodes are organized as clusters and each cluster has a head as the leader. All data from the sensor nodes within the cluster travel through the cluster head (see Figure 1.3).

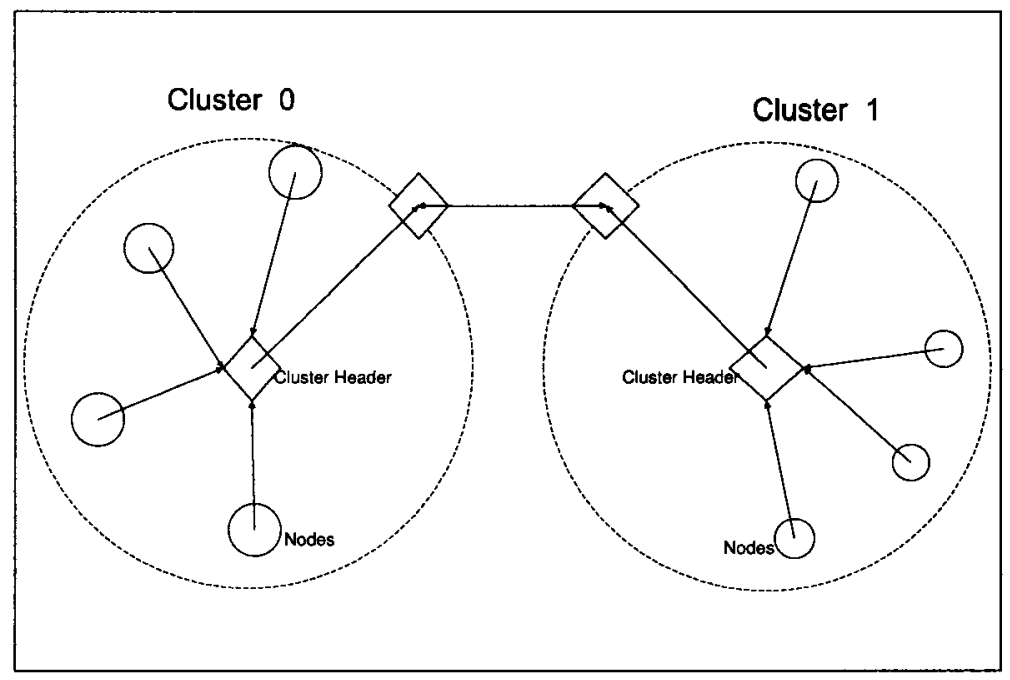

Figure 1.3: Clustered sensor networks with 12 nodes 


\subsubsection{Applications of wireless sensor networks}

WSNs have been useful in a variety of domains. The primary domains are as follows

\section{Environmental Observation}

WSNs can be used to monitor environmental changes, such as forest fires, soil erosion, air pollution and salt levels. One example could be water pollution detection in a lake that is located near a factory that uses chemical substances. Sensor nodes could be randomly deployed in unknown and hostile areas. They could relay the exact origin of a pollutant to a centralized authority so that appropriate measures can be taken to limit the spread of pollution. In this kind of application, the sensor nodes are usually distributed randomly. Due to the extreme size of the area and the application's complete coverage needs, a very large amount of sensors must be deployed. In this case, scalability becomes a crucial issue. Therefore, cluster techniques can be employed to achieve a more scalable system.

\section{Military Monitoring}

The military uses sensor networks for battlefield surveillance. Sensors could monitor vehicular traffic, track the position of the enemy, etc. 


\section{Structural Monitoring}

Sensors can be used in large structures such as buildings or factories to monitor climate changes. Thermostats and temperature sensor nodes can be deployed in all areas of the building. In addition, sensors can monitor vibration that could damage structure.

\section{Healthcare Applications}

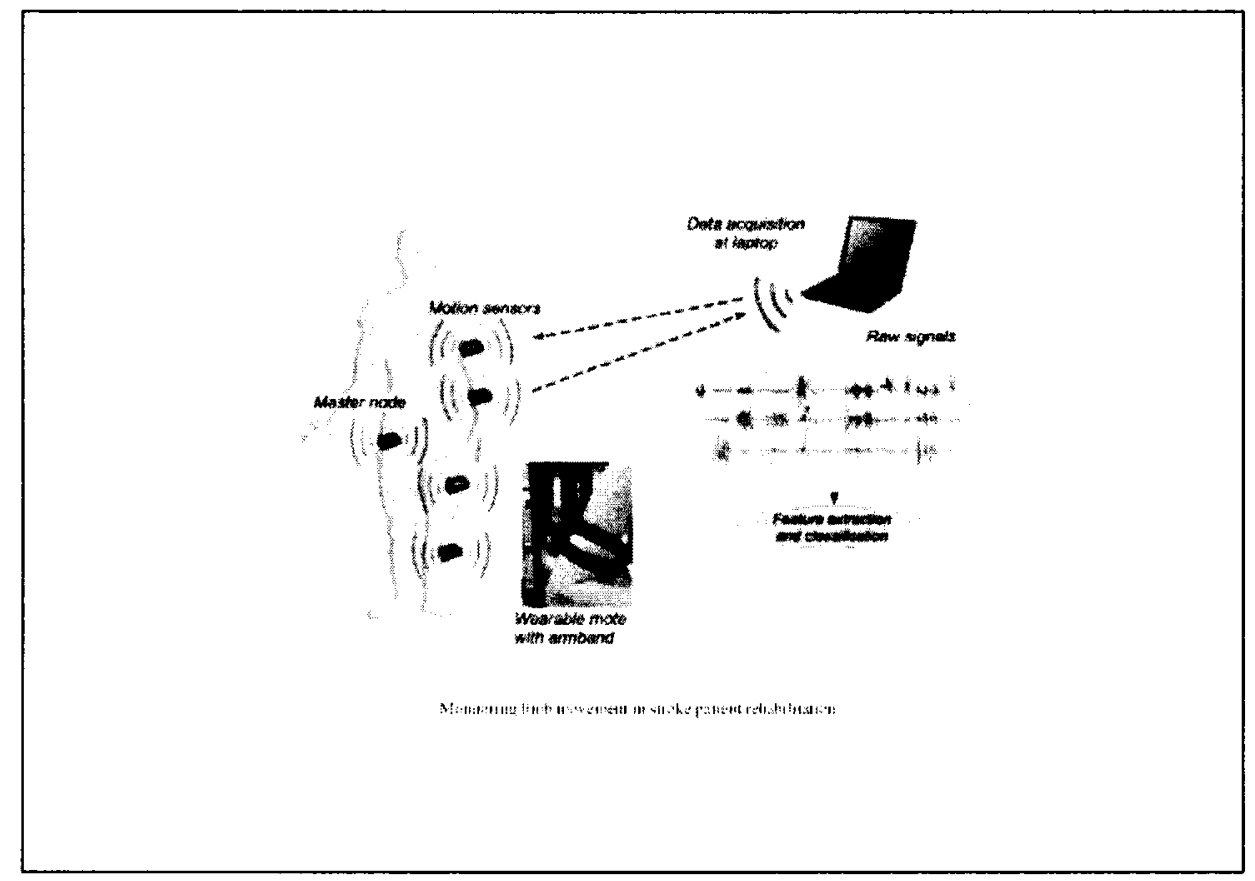

Figure 1.4: Healthcare application of WSNs

Sensors can be used for a range of medical applications, including pre-admittance and in-hospital emergency care, disaster response and stroke rehabilitation (see Fig- 
ure 1.4). Sensor networks allow data gathering and computation to be deeply embedded in the physical environment. This technology has the potential to impact the delivery and study of resuscitative care. It allows vital signs to be automatically collected and fully integrated into the patient care record, so they can be used for real-time triage, correlation with hospital records and long-term observation. A typical example is the Codeblue project at Harvard University [5].

\subsubsection{The need for congestion control in WSNs}

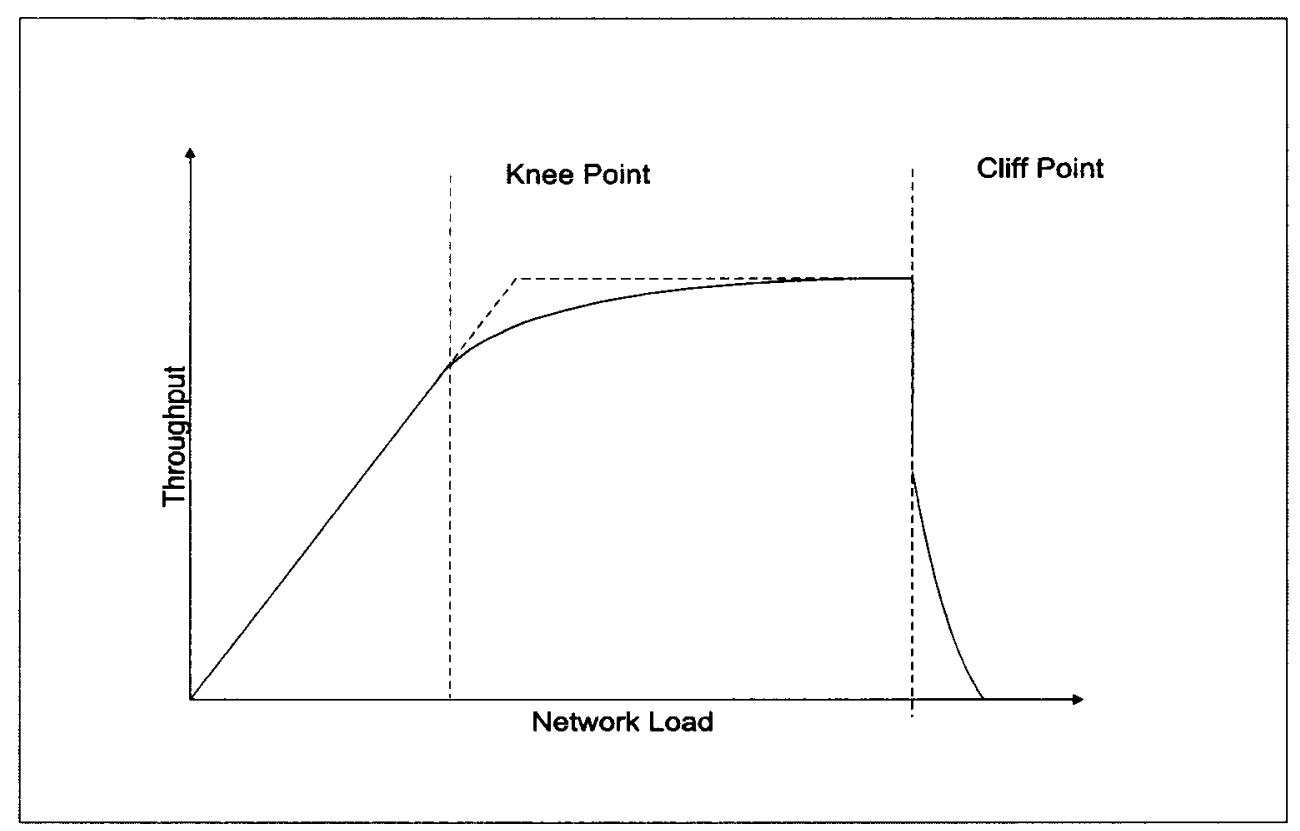

Figure 1.5: Network performance curve

Congestion usually occurs in the bottleneck of computer networks when sensor 
nodes receive more packets than they can send out. This mismatch of incoming and outgoing data rates results in congestion. In this situation, packets may be queued or dropped. As a result, throughput will degrade. Figure 1.5 shows network performance as a function of network load. At the beginning, network load is light, and throughput is linearly proportional to the load. When the load reaches network capacity (knee point), the throughput will not increase much. Packets will be queued, and the throughput may suddenly drop if packets are dropped due to buffer overflow (cliff point).

WSNs can be classified in two categories based on their data collection and transmission: event-based and dataflow-based. Both types produce convergent data flows from source nodes to base stations, which will potentially cause transmission congestion. In order to save the limited energy of source sensors and prolong the lifetime of the entire WSNs, congestion must be controlled.

\subsection{Congestion Control for Wireless Sensor Net- works}

In order to avoid congestion and improve network performance, a congestion control algorithm must be developed and used in a WSN. An ideal congestion control protocol wil make the network operate around the knee point and generally deals with: 
- Congestion detection in intermediate nodes

- Congestion notification between neighbors

- Rate adjustment in upstream neighbors

\subsubsection{Congestion detection}

Congestion detection plays an important role in congestion control. In TCP (transmission control protocol), congestion is detected at end nodes based on timeout or receiving three duplicate acknowledgments. In WSNs, the congestion detection mechanism usually depends on calculating packet service time [6], ratio between packet service time and packet inter-arrival time [7], or the queue length [8] [9] in an intermediate node. In this thesis, we adopt the congestion detection approach in [8] in which congestion is detected by monitoring the buffer space in the intermediate nodes.

\subsubsection{Congestion notification}

After detecting congestion, the congestion information is propagated to upstream nodes. The congestion information could be a congestion notification bit or other more rich information, such as allowable data rate or congestion degree which is a ratio of average packet service time over average packet inter-arrival time at each sensor node. There are two types of congestion notification approaches: 
- Explicit congestion notification

- Implicit congestion notification

In the explicit congestion notification mechanism, sensor nodes use special control packets to exchange congestion information. In the implicit congestion control notification approach, congestion information is piggybacked in the header of data packets. Thanks to the broadcast nature of wireless communication, neighbors can listen to nodes who are sending packets to get congestion information. The implicit notification approach avoids additional overhead, which is a good choice for wireless sensor networks due to the power constraint of WSNs. In this thesis, we used the implicit notification approach to exchange congestion information between neighboring nodes.

\subsubsection{Rate adjustment}

A rate adjustment mechanism can eliminate congestion, which in turn saves energy and prolongs the lifetime of WSNs. A number of rate adjustment mechanisms have been developed in last few years. A stop-and-start and hop-by-hop rate adjustment mechanism is used to eliminate congestion in [10]. In [9] and [11], an end-to-end and AIMD-like (Additive Increase Multiplicative Decrease) rate adjustment approach is employed. All of these rate adjustment mechanisms can only guarantee simple fairness instead of end-to-end weighted fairness. The priority-based congestion control proto- 
col (PCCP) [7] achieves end-to-end weighted fairness in single-path routing WSNs. In our work, we designed a priority-based rate adjustment algorithm called the Joint Priority Algorithm (JPA). The JPA guarantees end-to-end weighted fairness in multipath routing WSNs. In the JPA, intermediate nodes keep information about the joint priorities $(J P)$ of their neighbors. When congestion occurs in these nodes, the sending rates of upstream neighbors are automatically adjusted based on their joint priorities. In other words, when congestion occurs, upstream neighbors with important traffic will be allocated more bandwidth than others. However, when there is no congestion, each data source will send packets at the preset rate.

\subsection{Fairness Issues in Congestion Control}

The efficiency of a congestion control protocol depends on the following two performance objectives:

- Encrgy efficiency

- End-to-end fairness and end-to-end weighted fairness

Since sensor nodes have limited power, computational capacities and memory, energy efficiency is an important performance metric. A lot of previous work has been done in this regard. Another important performance metric is fairness. Congestion control protocols should guarantee fairness for different sensor nodes so that each sensor node 
can achieve fair throughput. If this does not occur, important information may be lost during transmission.

In real applications, sensor nodes may be either outfitted with different sensors or geographically deployed in different places. Therefore, they may have different source priorities and need to gain different throughputs. In this situation, weighted fairness is required so that sensor nodes can receive a throughput proportional to their source priorities. This concept will be defined in the following chapter.

\subsection{Previous Research Work and Problem State-}

\section{ment}

There are several congestion control protocols for WSNs. They differ in the way they detect and propagate congestion information and the way in which they adjust data rate when congestion occurs. In this section, we review some of these and discuss their limitations.

Congestion detection and avoidance (CODA) [9] proposes an open-loop, hop-byhop backpressure mechanism and a closed-loop, multi-source regulation mechanism in event-driven WSNs. Sensor nodes detect congestion by monitoring channel utilization and buffer-occupancy levels. In response to congestion, the congested sensor nodes send backpressure messages to their neighbors which may drop packets, reduce their 
sending rate and further propagate backpressure messages. If the sending rate of a source node is greater than the preset threshold, the source node must receive a continuous stream of ACKs from the base station in order to maintain that rate. By this means, the base station is able to limit the sending rate of a source node based on deciding how many ACKs to broadcast. CODA employs the AIMD (Additive Increase Multiplicative Decrease) coarse rate adjustment. It only guarantees simple fairness when congestion occurs.

The event-to-sink reliable transport protocol (ESRT) [12] monitors local buffer levels in intermediate sensor nodes and sets a congestion notification bit in the packet when the buffer overflows. If a base station receives a packet whose congestion notification bit is set, it broadcasts a control signal to inform all source nodes to reduce the sending rate according to certain proportion. ESRT limits the sending rate of all source nodes when congestion occurs, regardless of where the hot spot takes place in WSNs. The most effective way is to regulate the source nodes that are responsible for this congestion.

The priority-based congestion control protocol (PCCP) [7] defines a new variable congestion degree as a ratio of average packet service time over average packet interarrival time at each sensor node. The congestion degree is intended to reflect the current congestion level of each sensor node. Based on the congestion degree, PCCP employs a hop-by-hop rate adjustment technique called priority-based rate adjust- 
ment (PRA). PRA adjusts the scheduling rate and the source rate of each sensor node in a single-path routing WSN.

In the tree-based network topology of single-path routing WSNs, a sensor node will have only one downstream neighbor, but it may have multiple upstream neighbors. The whole data flow generated by a source node will pass through the nodes and links along with the single routing path. Sensor nodes learn the number of upstream data sources in the subtree roots and measure the maximum downstream forwarding rate. Finally, they will calculate the per-source rate based on the priority index of each source node. For example, in Figure 1.6, we assume all the nodes are source nodes and their priority index equals 1 . Therefore the number of data sources coming from link $\left(i_{1}, j_{1}\right)$ is 5 , while the number of data sources coming from link $\left(i_{2}, j_{1}\right)$ is 2 . In response to the congestion in node $j_{1}$, the sending rate of the upstream link $\left(i_{1}, j_{1}\right)$ is regulated to $\left(\frac{5}{5+2}\right) \times r_{s v c}^{i}$, while the sending rate of the upstream link $\left(i_{1}, j_{1}\right)$ is limited to $\left(\frac{2}{5+2}\right) \times r_{s v c}^{i} . r_{s v c}^{i}$ is defined as the scheduling rate of node $j_{1}$.

The priority index is applied as a coefficient of priority which reflects the importance of each source node. However, this algorithm will work well only in single-path routing WSNs. In a multi-path situation, the packets of a flow may be routed on many paths and only one fraction of a flow will pass through a particular node or link. It is difficult to calculate the sum of the fractions of all data flows which pass through that node or link using the algorithm in PCCP. 


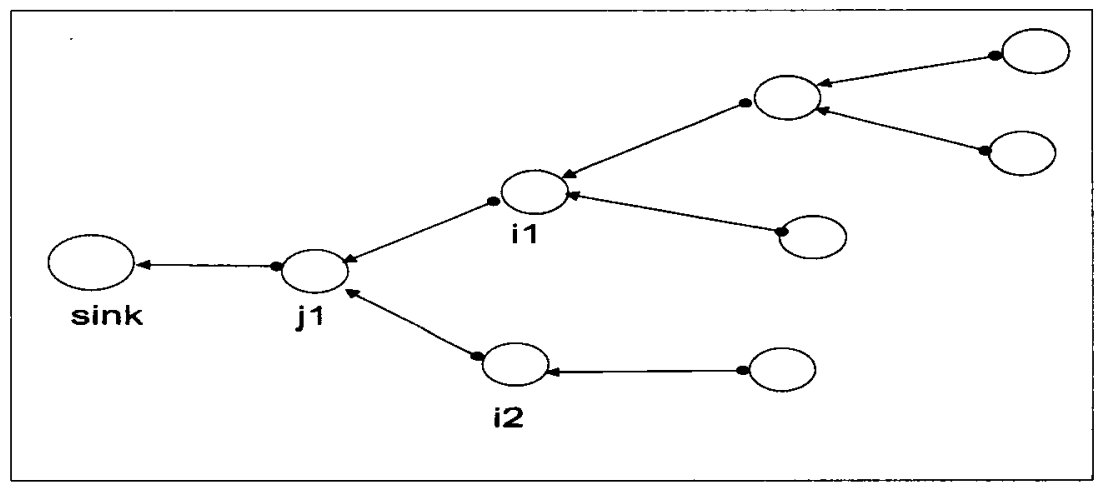

Figure 1.6: Tree-based network model in single-path routing WSNs

Other studies have examined the multi-path routing issue, but they do not consider the fairness problem. In this thesis, we propose a joint priority algorithm which guarantees weighted fairness in multi-path routing WSNs.

\subsection{Objectives of this Thesis}

Most current research in congestion control protocols result in extremely biased bandwidth allocations. Fairness problems must be carefully addressed in most WSNs. It also is a challenge to design a fully distributed fairness solution due to a lack of global knowledge about the distribution of data sources and their routing paths. In prior studies, only simple fairness, in which each sensor node reduces reporting rates uniformly when congestion occurs, is guaranteed, or weighted fairness is achieved only in single-path routing WSNs. Those kind of congestion control protocols limited the 
applications of WSNs.

This thesis presents a new rate adjustment algorithm to achieve weighted fairness in multi-path routing WSNs. In our algorithm, it is assumed that the set of base stations (sinks) are static and the location of data source is fixed.

The main focus of this thesis is the design of priority-based congestion protocols and the performance analysis of the proposed joint priority-based congestion control protocol. This thesis has three objectives:

1. To propose a rate adjustment algorithm that will eliminate congestion for multipath routing WSNs, and guarantee end-to-end weighted fairness.

2. To design and implement simulation programs to evaluate the performance of the proposed algorithm.

3. To analyze the simulation results and present valid conclusions regarding the proposed models.

\subsection{Thesis Contributions}

This thesis proposes a new congestion control algorithm for wireless sensor networks. The proposed algorithm guarantees end-to-end weighted fairness in multi-path routing WSNs. 
In order to evaluate its performance, the proposed new congestion control algorithm is compared with two previous schemes. It is then confirmed by simulations that our algorithm demonstrates improved fairness performance. Priority-based end-toend fairness is achieved among competing data flows. In addition, this new algorithm is able to work with any routing protocol.

\subsection{Thesis Organization}

The remainder of this thesis is organized as follows:

Chapter 2, "Background", provides a brief introduction to the Carrier Sensing Multiple Access (CSMA), the Performance Metrics of Congestion Control protocol, the Rate Adjustment Algorithm, and the Routing Protocol in WSNs, which are all relevant to the design of congestion control protocols.

Chapter 3, The "Joint-priority Algorithm" focuses on investigating the algorithm that defines an inventable node and network model, proves that the joint priority of each node or link can be calculated using local information and describes the main features of the joint-priority algorithm.

Chapter 4, "Simulation Models and Results Analysis" describes the simulation model and the simulation results. In this chapter we also introduce evaluation criteria and evaluate the proposed algorithm against other congestion control protocols under 
different scenarios. Finally, the benefits of our proposed algorithm are explained.

Chapter 5, "Conclusions" contains a summary of the key findings and some suggestions for further improvements and future research directions. 


\section{Chapter 2}

\section{BACKGROUND}

Wireless sensor networks (WSNs) detect signals in the environment and transfer these signals to the end user via wireless communication channels. In this chapter, we will describe some important telecommunication technologies that have been used in wireless sensor networks for congestion control.

\subsection{Carrier Sensing Multiple Access (CSMA)}

In WSNs, nodes within the same transmission range share a single transmission medium. Some kind of medium access control protocol is needed in order to avoid collision and better utilize bandwidth. Carrier sensing multiple access (CSMA) is a random access protocol used on shared network topologies such as WSNs. CSMA 
allows users to transmit data in a non-predetermined way. The sensor nodes attach to the network channel listening (carrier sense) before transmitting. Multiple access (MA) indicates that many nodes can connect to and share the same transmission medium. If the channel is in use, the node will wait for a random period and re-sense. A random period is used to minimize collision since other users may also want to access the medium at the same time. Once the channel is idle, the node can start transmission. All nodes have equal access to the network when it is clear.

Even though nodes attempt to sense before sending packets, there is a good chance that two nodes will attempt to access at the same time. The transmission time between one end of the channel and another is enough that one node may access the channel even though another may have already just accessed it. CSMA/CA (carrier sense multiple access/collision avoidance) is used to avoid this kind of collision. The basic idea of CA is to prevent collisions at the moment they are most likely to occur. Before any real data is transmitted, the sensor node has to broadcast a signal onto the network in order to listen for collision scenarios and to tell other nodes to stop broadcasting.

Another issue for wireless networks is the problem of hidden/exposed terminals. As show in Figure 2.1, when node N1 is transmitting a packet to node N2, node N3 cannot sense the transmission from N1 since N1 and N3 cannot hear each other. N3 may transmit a packet to $\mathrm{N} 2$ and cause a collision at $\mathrm{N} 2$, which is called a hidden ter- 


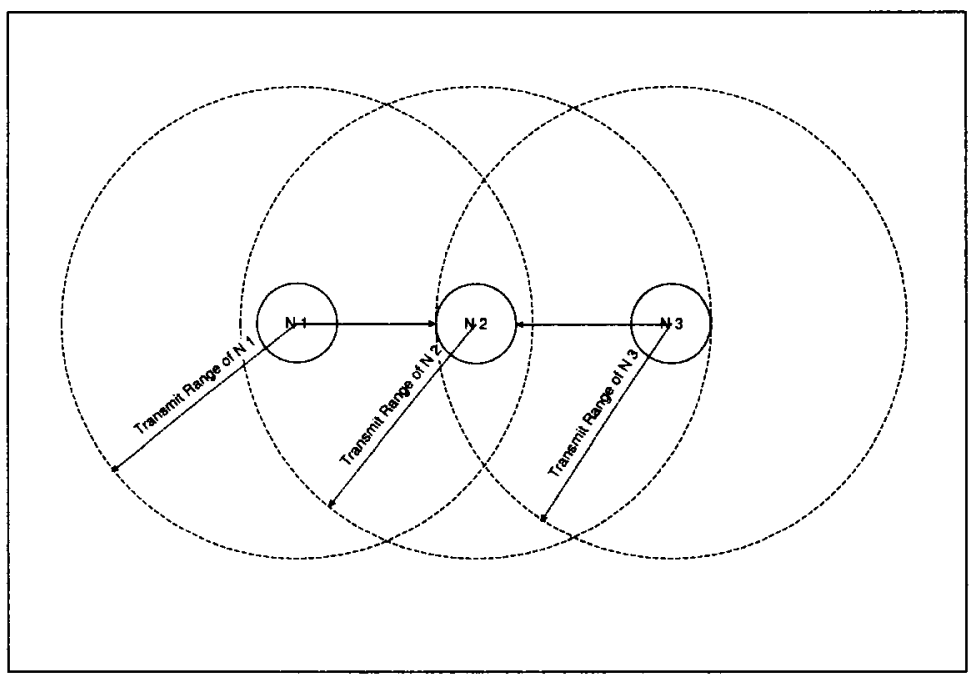

Figure 2.1: Hidden terminal problem

minal problem because N1 is hidden from N3. The exposed terminal problem occurs when a node is prevented from sending packets to other nodes due to a neighboring transmitter. For instance, in Figure 2.2, node N3 cannot start a transmission to N4 when $\mathrm{N} 2$ is sending a packet to N1, because N3 considers after carrier sense that it will interfere with the transmission by its neighbor node N2. However N4 could still receive the transmission from $\mathrm{N} 3$ without interference since it is out of range from N2. This is exposed terminal problem since N3 is exposed to N2.

An RTS/CTS mechanism can be used to solve hidden/exposed terminal problems. For example, a node sends a Request-To-Send (RTS) message before sending real data packets. The receiving node replies with a Clear-To-Send (CTS) message. Any other nodes within this community receiving the RTS but not CTS should defer to allow 


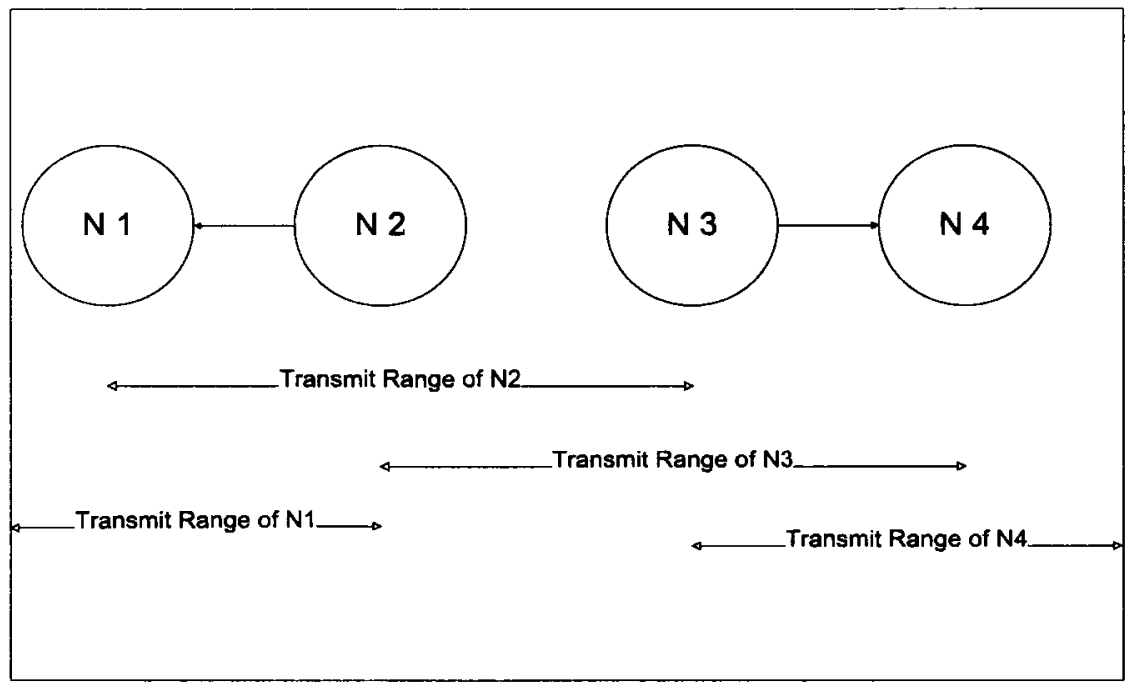

Figure 2.2: Exposed terminal problem

CTS which means they can transmit to other neighboring nodes. In this way, the exposed terminal problem will be solved. Other nodes receiving the CTS should defer to allow the data which means they must refrain from sending data for some time. This will solve the hidden terminal problem.

Figure 2.3 provides an example. When node N2 wants to send a data packet to node N3, it first sends a Request-To-Send (RTS) message to node N1 and N3. When node N3 receives the RTS message, it broadcasts a Clear-To-Send (CTS) message to node N2 and N4. As node N2 receives the CTS, it starts to transmit the data packets. However N4 will defer its data transmission upon receiving the CTS since it knows $\mathrm{N} 3$ will receive data from some nodes, which solves the hidden terminal problem. N1 will still be able to send the data to other nodes when it receives the RTS but not 


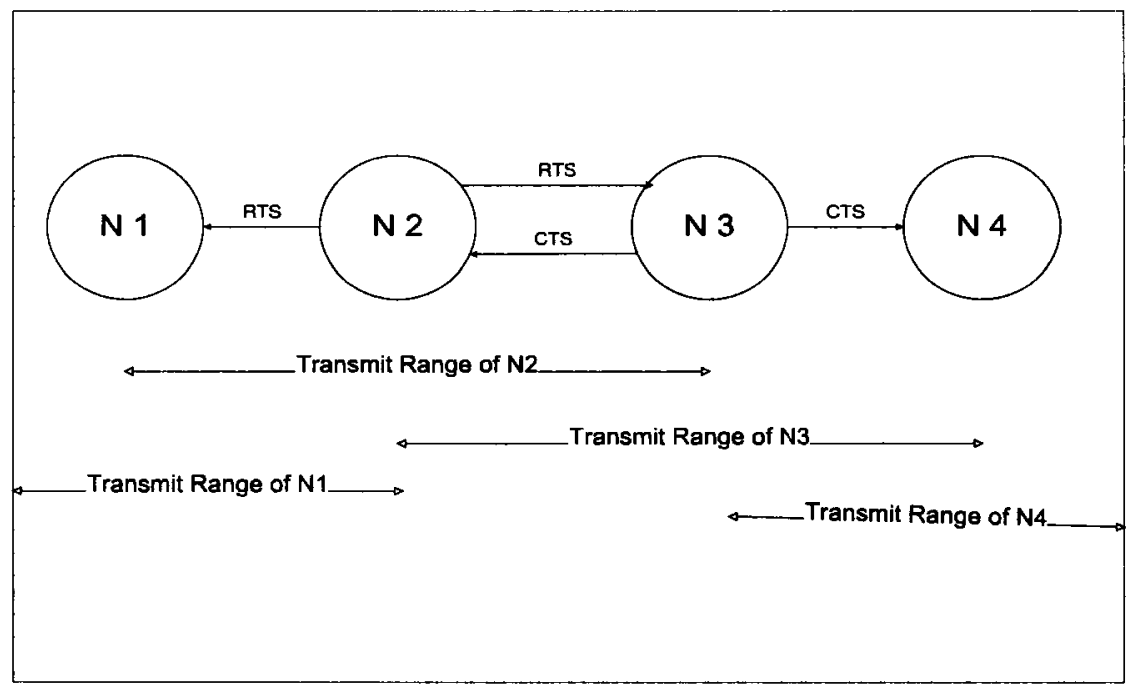

Figure 2.3: RTS/CTS protocol

CTS because it knows the destination node is not in its transmission range.

\subsection{Rate Adjustment Algorithm}

\subsubsection{Additive increase multiplicative decrease (AIMD)}

A sensor node can adjust its sending rate when it receives a congestion notification. AIMD (Additive Increase Multiplicative Decrease) is employed in most of congestion control protocols. AIMD provides a feasible linear control algorithm [13]. The 
algorithm is represented by

$$
\lambda_{t+1}=\left\{\begin{array}{ccc}
a_{I}+\lambda_{t} & : & \text { Increase } \\
b_{D} \cdot \lambda_{t} & : & \text { Decrease }
\end{array}\right.
$$

where $\lambda_{t+1}$ and $\lambda_{t}$ are the sending rate at time point $t+1$ and $t$, and $a_{I}$ and $b_{D}$ are parameters which satisfy

$$
\begin{gathered}
a_{I}>0 \\
1>b_{D} \geq 0
\end{gathered}
$$

AIMD is implemented as either a window-based or a rate-based mechanism. In window-based AIMD, the outstanding packets of the sender are limited. Rate-based AIMD regulates the sending rate at which the packets can be sent out. In the Internet, TCP(transmission control protocol) employs the window-based AIMD mechanism. TCP increases the window by one packet every round-trip time (RTT) if no packet is lost and decreases the congestion window by half every RTT if a packet is lost. In heavy congestion, for example, when a retransmitted packet is itself dropped, TCP uses a retransmit timer, which is exponentially backed off if repeated losses occur. This situation is depicted in Figure 2.4.

A dynamic model was built to analyze the rate-based AIMD mechanism [14]. In Figure 2.5, $\lambda(t)$ is the source sending rate and $q(t)$ is the bottleneck's queue size at 


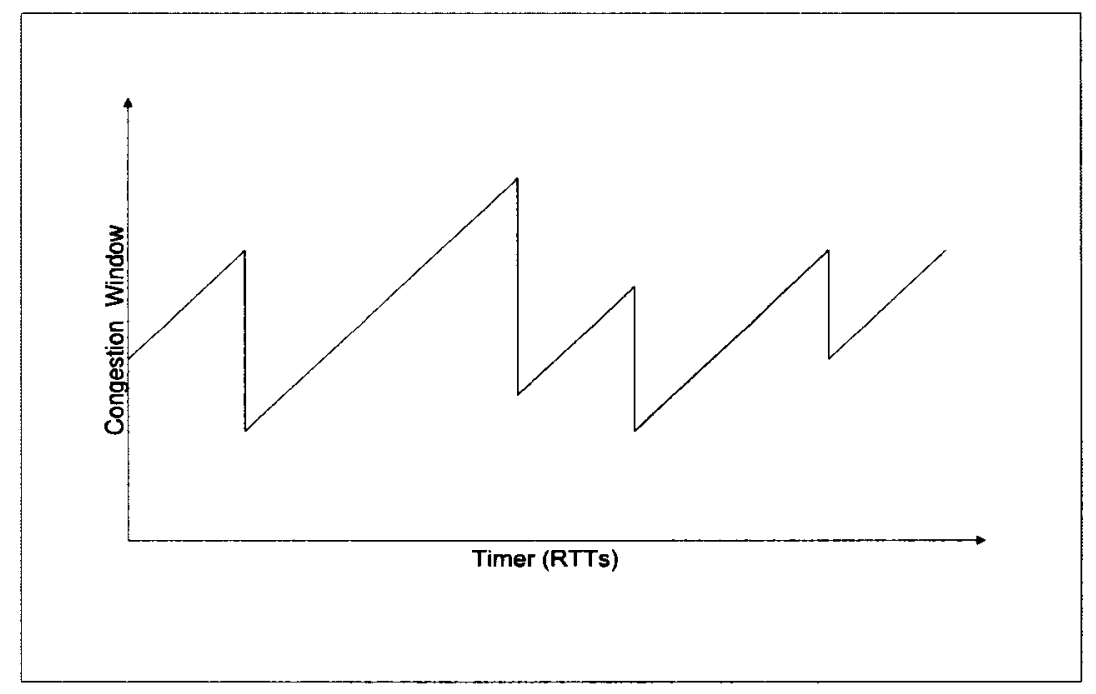

Figure 2.4: TCP AIMD Mechanism

time $t . \mu$ is the bottleneck's service rate. $\tau_{F}$ is the propagation delay from the source to bottleneck, while $\tau_{R}$ is the propagation delay from bottleneck to the source. $\lambda(t)$ and $q(t)$ are the derivatives of $\lambda(t)$ and $q(t)$. They are given by Eq. (2.3) and Eq. (2.4) Where, $\alpha$ is the linear increasing rate and $\beta$ is the time constant of the exponential decreasing curve.

$$
\dot{q(t)}=\left\{\begin{aligned}
0 & \text { if } q(t)=0 \text { and } \lambda\left(t-\tau_{F}\right)<\mu \\
\lambda\left(t-\tau_{F}\right)-\mu & \text { otherwise }
\end{aligned}\right.
$$




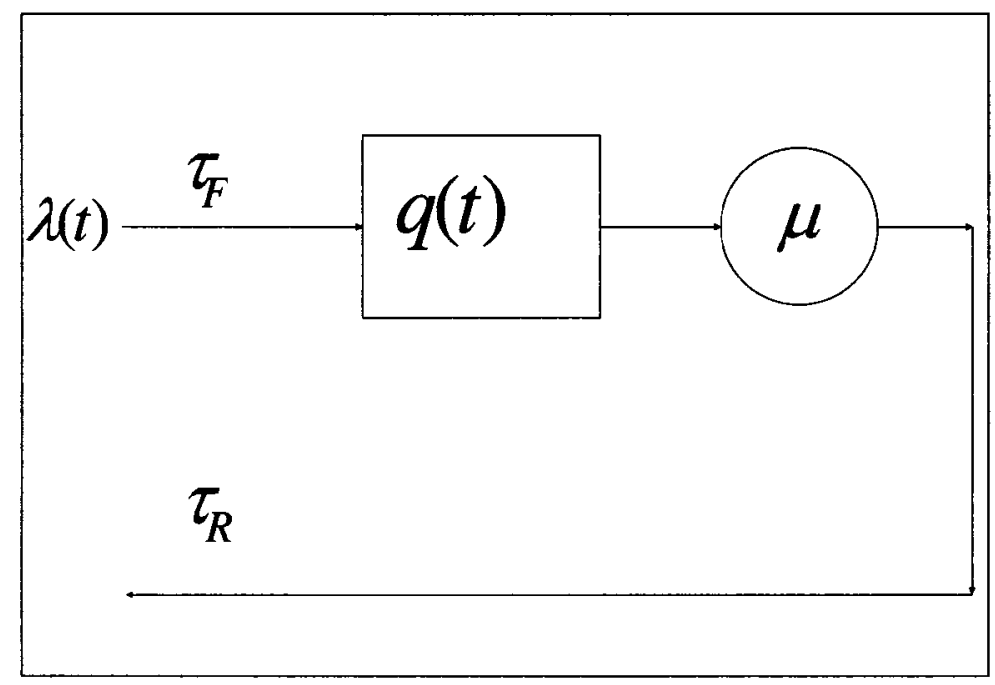

Figure 2.5: Rate-based AIMD Model

$$
\dot{\lambda(t)}=\left\{\begin{aligned}
\alpha & \text { if } q\left(t-\tau_{R}\right)=0 \\
-\frac{\lambda(t)}{\beta} & \text { if } q\left(t-\tau_{R}\right)>0
\end{aligned}\right.
$$

\subsubsection{Token bucket algorithm}

The token bucket is a control mechanism that decides when traffic can be transmitted based on the presence of tokens in a bucket which is used to control the amount of data that is injected into a network. The token bucket contains tokens, each of which can represent a unit of bytes or a single packet. In Figure 2.6, the token is generated at a rate of $r$ and $b$ is the size of the bucket. The data flows come from the left, while 


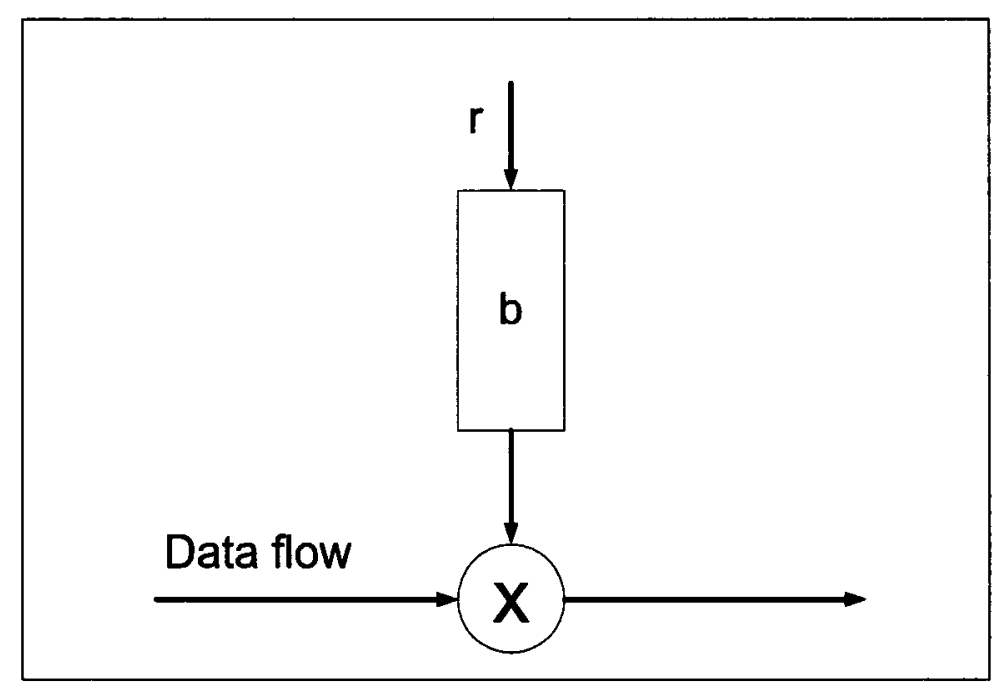

Figure 2.6: Token bucket model[15]

the token flows go into the bucket from the top. When the bucket is full of tokens, new tokens are thrown away. When a packet arrives from the left, if the number of tokens is at least equal to the number of bytes in the packet, the packet may be transmitted. Otherwise, if there are not enough tokens in the bucket, the packets must wait until there are enough[15].

The token bucket algorithm can be understood as follows:

- A token is added to the bucket every $1 / r$ seconds.

- If $b$ is the bucket size, then the bucket can hold at the most, the number of $b$ tokens. If a token arrives when the bucket is full, it is discarded,

- If more than $n$ tokens are available when a packet of $n$ bytes arrives, $n$ tokens 
will be removed from the bucket and the packet will be sent to the network. Otherwise, no tokens are removed from the bucket and the packet will be considered non-conformant.

\subsection{Routing Protocol in WSNs}

Energy dissipation is directly related to underlying routing techniques. Depending on the application requirements, different routing strategies may be implemented on wireless sensor networks. Routes may form tree structures where roots are sink nodes, or similarly, multi-path routing strategies may also be employed when communication links are less reliable. In this section, we will describe routing technologies in wireless sensor networks.

\subsubsection{Challenges and design issues in WSNs routing protocol}

Routing in WSNs is very challenging because of the inherent characteristics that distinguish it from other networks. First of all, a large number of sensor nodes will make it impossible to implement IP-based routing protocol in WSNs because the overhead of ID address maintenance is very high. Secondly, the sensor nodes may be constrained in terms of energy, computing and storage capacities. Therefore, careful resource management is required. Thirdly, the information collected by the sensors may have 
some redundancy. This redundancy must be exploited by the routing protocol in order to conserve the energy. Compared to address-centric networks, sensor networks are called data-centric networks. In data-centric networks, a set of attribute-value pair queries is used. Fourthly, due to the many-to-one convergent data flow nature, there is a high probability that congestion will happen near the base station. Finally, in some sensor networks applications, the position of the sensor nodes is important since data collection is usually based on location. Some protocols approximate the position of sensor nodes based on radio strength. Using GPS technology is also a good choice for the location problem, but its cost is high.

Routing protocols in WSNs must be carefully designed because of these differences. The main design goals of routing protocols in WSNs is to collect and transmit data while trying to prolong the lifetime of the network. This process is affected by the following challenging factors [16]:

- Energy saving without losing accuracy: Sensor nodes use their limited energy to perform computation and data transmission in a wireless environment. Energy efficient routing protocols are essential. In multi-hop WSNs, the intermediate nodes relay data packets towards the data source. Therefore, the failure of a node may cause significant topological changes and reroute data packets.

- Fault tolerance: The failure of a particular node should not affect the overall task of the sensor network. When the failure of nodes occurs, routing protocols 
must adjust transmit powers and signaling rates on the existing links to reduce energy consumption. On the other hand, routing protocols will reroute data packets through other links where more energy is available.

- Scalability: Hundreds or thousands of sensor nodes are deployed in the sensing area. Routing protocols must be able to work with this huge number of sensor nodes.

- Coverage: Each sensor node can only cover certain areas of the environment. Coverage is limited by the transmission range of sensor nodes.

- Transmission media: In wireless sensor networks, sensor nodes are linked by wireless communication mediums. The traditional problems of wireless channels, such as high error rates, will also affect routing protocol design in WSNs.

- Data aggregation: Due to the possibility that sensor nodes may generate significant amounts of rcdundant data, data packets from different nodes should be aggregated in order to reduce the number of transmissions. Data aggregation has been used to achieve energy efficiency and data transfer optimization in different routing protocols. 


\subsubsection{Classification of routing protocol in WSNs}

In this section, we will review state of the art routing protocols in WSNs. Based on network structure, the routing protocols in WSNs are classified into flat-based routing protocols, hierarchical-based routing protocols and location-based routing protocols. Routing protocols can be classified into multipath-based routing, querybased routing, negotiation-based routing and coherent-based routing according to protocol operation. Figure 2.7 shows the classification of routing protocols in WSNs.

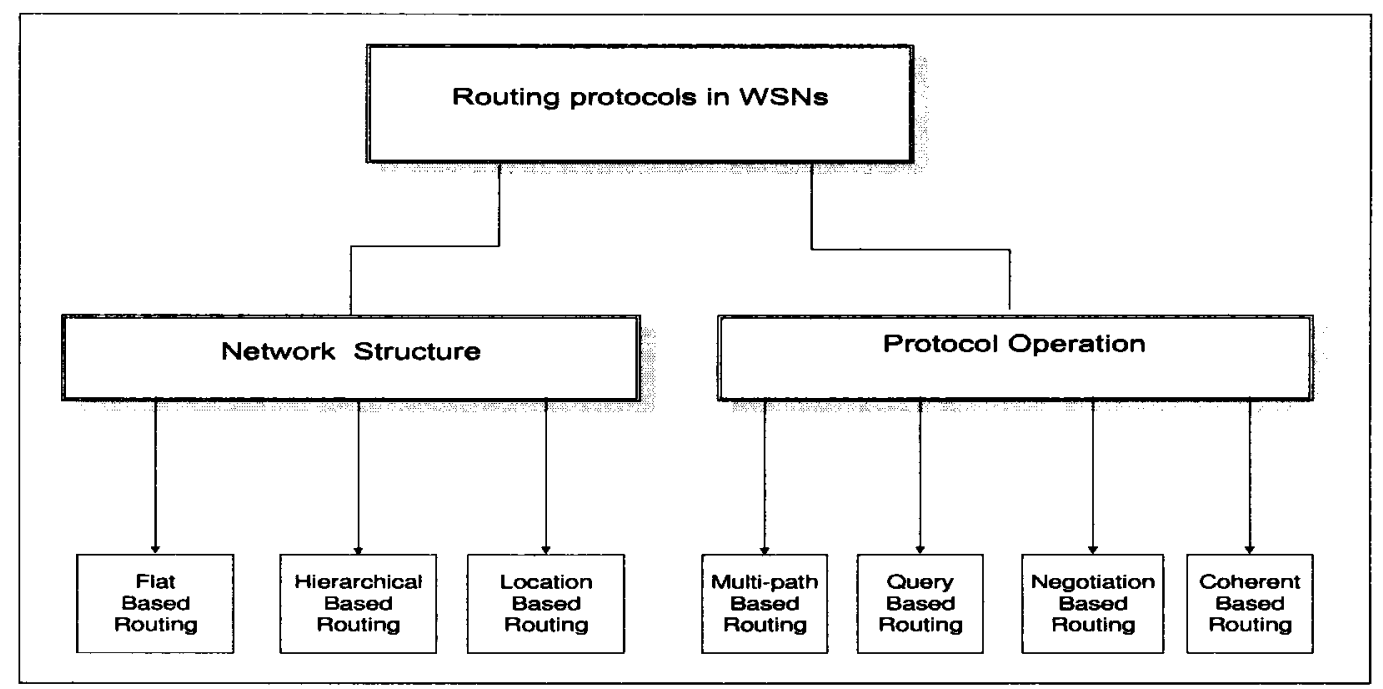

Figure 2.7: The classification of routing protocols in WSNs 


\section{Flat-based Routing Protocols}

In this type of routing protocol, all nodes play equal roles and collaborate together to perform the sending task. Due to the large number of such nodes, data centric routing is introduced instead of address centric routing. Sensor nodes in certain regions send data packets to the base station based on attribute-based queries from the base station. Adaptive protocols for information dissemination in wireless sensor networks [17] and directed diffusion [18] are two routing protocols that use the data centric routing. [19],[20] and [21] also belong to flat-based routing.

\section{Hierarchical-based Routing Protocols}

The nodes in this routing protocol are assigned different roles or functionalities. One typical hierarchical-based routing protocol is cluster-based routing. Within a cluster, the cluster heads have more energy and are used to process and send information, while other low energy nodes within a cluster can be used to sense targets. By performing data aggregation and fusion, hierarchical based routing protocols lower energy consumption and prolong the lifetime of the whole sensor network. There are mainly two layers in WSNs hierarchical based routing. One layer is used to select the cluster head, while another is used to route data packets. In [22], a hierarchical clustering algorithm was introduced, which is called low energy adaptive clustering hierarchy (LEACH). 
LEACH randomly chooses cluster heads and rotates the role among sensors in the networks in order to avoid draining the battery of certain sensor nodes. This evenly distributes the energy load and prolongs the lifetime of networks. The cluster heads compress the data coming from the same cluster and sends the aggregated data packets to the base station in order to reduce the number of transmissions.

The operation of LEACH consists of two phases: the setup phase and the steady state phase. During the setup phase, a cluster is organized and cluster heads are selected. In the steady state phase, real data transmission to the base station occurs. After a cluster is created, sensor nodes in the cluster should decide whether or not they will become a cluster head. This decision is made by node $n$ by choosing a random number between 0 and 1. If the number is less than the threshold $T(n)$, the node $n$ is considered to be a cluster head. The threshold $T(n)$ is decided by the following equation:

$$
T(n)=\left\{\begin{aligned}
\frac{p}{1-p \times(r \bmod (1 / p))} & \text { if } n \in G \\
0 & \text { otherwise }
\end{aligned}\right.
$$

$p$ is the desired percentage of cluster heads and $G$ is the set of nodes that are involved in cluster head election. New cluster heads will broadcast a advertisement message to the nodes of this network. All of the non-cluster head nodes decide on the cluster to which they want to belong after receiving the advertisement message. 
In the steady state phase, non-cluster head nodes sense and transmit data to cluster heads. Cluster heads aggregate these data before sending them to the base station. After that, the networks go back into another round of selecting new cluster heads and enter the setup phase again. Other hierarchical-based routing protocols include [23], [24],[25] and [26].

\section{Location-based Routing Protocols}

In location-based routing protocols, sensor nodes are addressed by their positions. They figure out the positions of their neighbors depending on incoming signal strengths. [27] is a typical example. In order to save energy, sensor nodes go to sleep if there is no activity. Some nodes are selected as coordinators based on their position. Nodes which are selected as coordinators stay awake and perform the routing task in the sensor networks, while non-coordinator nodes remain in energy saving mode. The non-coordinator nodes decide whether to wake up and become coordinators if two neighbors of a non-coordinator node cannot reach each other directly. SPAN defines a random backoff delay to prevent simultaneous decision making. The delay is a function of the number of other nodes in its neighborhood that can be bridged using this node and the amount of the energy that remains.

$$
\text { delay }=\left(\left(1-\frac{E_{r}}{E_{m}}\right)+\left(1-\frac{C_{i}}{\left(\frac{N_{i}}{2}\right)}\right)+R\right) \times N_{i} \times T
$$


Where $E_{r}$ is the amount of energy at a node that still remains, while $E_{m}$ denotes the maximum amount of available energy at the same node. Let $N_{i}$ be the number of neighbors of node $i$ and $C_{i}$ be the number of additional pairs of nodes among these neighbors that can be connected if node $i$ becomes a coordinator. $T$ is the round-trip time of a small packet over a wireless link. $R$ is the random number picked from interval $[0,1]$. Other location-based routing protocols include [28], [29] and [30].

\section{Multi-path Routing Protocols}

In single path routing protocols, the periodic, low rate flooding of events enables local re-routing around failed nodes. Due to energy efficiency which is very important in wireless sensor networks, such flooding can adversely impact network lifespan. Accordingly, it is desirable to find alternative techniques to provide greater resilience if failures occur. In this section, we study routing protocols that use multiple paths rather than a single path in order to enhance network performance. Multi-path routing allows the establishment of multiple paths between source and destination. There are two reasons why multi-path routing is introduced. The first one is load balancing. The traffic between the source-destination pair is split across multiple partially or completely disjoint paths. The second use of multipath routing is to increase the likelihood of reliable data delivery. In this approach, multiple copies of data are sent along different paths, allowing for resilience to failure of a certain number of paths. Load balancing can spread energy utilization across nodes in WSNs. 
Duplicate data delivery along multi-path can result in more accurate tracking in surveillance applications but increases energy expense.

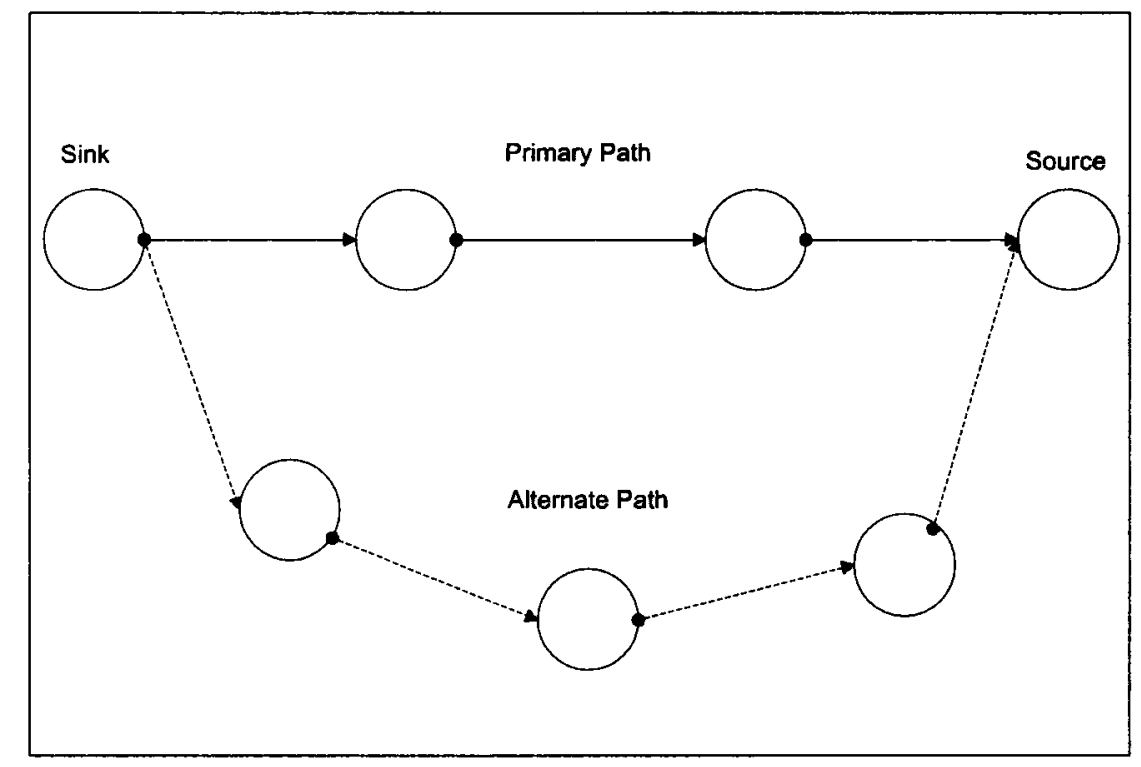

Figure 2.8: Node-disjoint multipath routing

There are two different approaches to constructing multipath between the source and destination. One is complete node-disjoint multipath routing shown in Figure 2.8, where alternate paths do not intersect the original path or each other. This approach is useful for delivering data in unreliable environments [31]. It is known that network reliability can be increased by providing several paths from source to destination and sending the same packet on each path. However, if this approach issured, traffic will increase significantly. Hence, there is a tradeoff between the amount of traffic and the reliability of the network. 


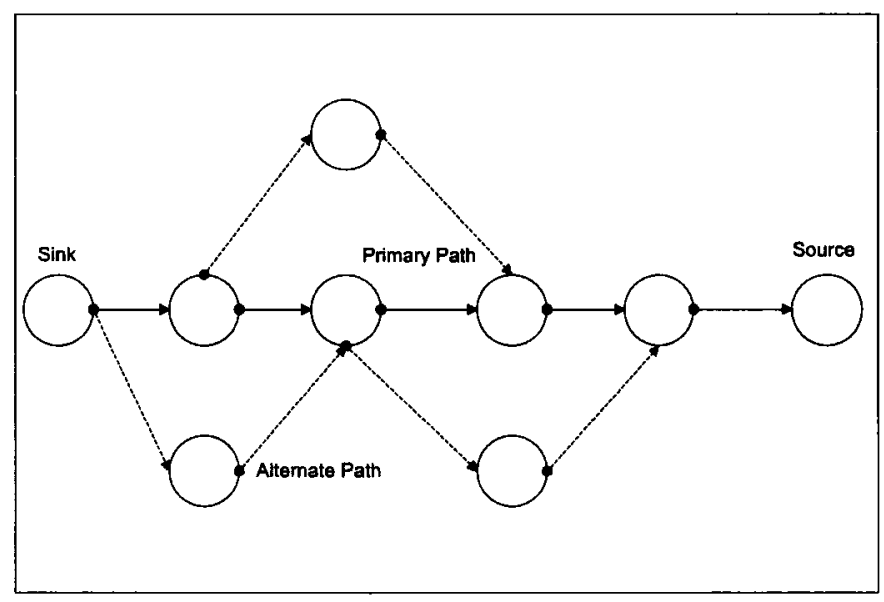

Figure 2.9: Braided multipath routing

Another approach shown in Figure 2.9 builds many braided paths. With braided paths, there are typically no completely disjoint paths but many partially disjoint alternate paths. Directed diffusion [18] is a good candidate for a more robust multipath routing which builds many braided paths instead of completely disjoint paths. Based on the directed diffusion paradigm, a multipath routing scheme that finds several partially disjoint paths is studied. The reason for using these braided paths is to keep the cost of maintaining the multipath low. The cost of alternate paths is comparable to the primary path because they tend to be much closer to the primary path. Other multi-path Routing Protocols include [32] [33][34][35]. 


\section{Query-based Routing}

In query-based routing, destination nodes send a query for data. A node with this data sends data which matches the query back to the nodes which initiated the query. Directed diffusion [18] and [36] are two examples of this type of routing. The base station sends out the interest messages to sensors. As the interest is propagated throughout the sensor network, gradients from the source back to the base station are set up. When the source nodes have data for the interest, they send the data along the interests gradient path.

\section{Negotiation-based routing}

The use of flooding to disseminate data will produce implosion and overlap in the sent data. As a result, nodes will receive duplicate copies of the same data. Negotiationbased routing in [37] is designed to disseminate the data of one sensor to all other sensors assuming these sensors are potential based-stations. Hence, the main idea of negotiation-based routing in WSNs is to suppress duplicate information and prevent redundant data from being sent to the next sensor or the base station by conducting a series of negotiation messages before the real data transmission begins. 


\section{Coherent-based Routing}

Routing techniques employ different data processing techniques before the nodes send receiving packets to another nodes. Two main data processing techniques are coherent processing and non-coherent processing. In non-coherent data processing routing, nodes will locally process raw data before it is sent to other nodes for further processing. In coherent routing, the data is forwarded to aggregators that perform further processing after minimum processing. Minimum processing typically includes tasks like time stamping, duplicate suppression, etc. In order to perform the energy-efficient routing, coherent processing routing is normally selected in WSNS.

\subsection{Performance Metrics of Congestion Control Pro- tocols in WSNs}

There are two mian causes of congestion in WSNs. One is due to packet arrival rate exceeding packet service rate, which is called node level congestion. Another type of congestion is link level congestion which is caused by issue with link level performance, such as contention and interference. Congestion in WSNs can cause buffer overflow which in turn leads to longer queuing time and more packet loss. Not only will packet loss reduce reliability, it will also waste limited energy and lower energy efficiency. Link level congestion will also result from transmission collisions. For example, if 
CSMA is used to share radio resources.

The performance of a congestion control protocol can be evaluated using metrics such as:

- Energy efficiency

- End-to-end fairness and end-to-end weighted fairness

\subsubsection{Energy efficiency}

In WSNs, sensor nodes usually have limited energy. Energy and power constraints will significantly affect the problem of congestion control protocol in wireless sensor networks. It is important that congestion control protocol maintains high energy efficiency in order to maximize entire system lifespan. Therefore, one of the metrics to assess the performance of the congestion control protocol should be energy efficiency.

The total energy that a sensor node spends on delivering one packet to its final destination will include any retransmission necessary. A wireless sensor node typically consists of three basic states:

- Idle state

- Transmitting state

- Receiving state 
Energy for transmission dominates total energy cost. There are three factors that must be carefully evaluated when we design congestion control protocol for WSNs. These factors include the number of retransmissions, overhead of control messages and transmission distance. In [10], the energy efficiency is defined as the number of hops useful packets travel, divided by the total number of packet transmissions in the network. A useful packet is any packet that eventually reaches the base station.

$$
\eta=\frac{\sum_{u \in U} h o p s(u)}{\sum_{p \in P} \sum_{h \in h o p s(p)} x m i t s(p, h)}
$$

where $U$ is the set of useful packets, and $u \in U$, which is considered as a useful packet. $P$ is the set of all packets and $p \in P$ which is a particular packet. hops $(p)$ ranges over each hop that packet $p$ takes and $x$ mit $(p, h)$ counts the number of transmissions that packet $p$ undergoes at hop $h$. This efficiency metric reflects failed transinission, buffer drop and retransmission due to channel loss.

\subsubsection{End-to-end fairness and end-to-end weighted fairness}

Fairness can be defined in many different ways. We consider the network to be fair if the number of packets received from each source in the network is approximately the same over a period of time. In wireless sensor networks, sensor nodes are scattered in a geographical area. Due to the many-to-one convergent nature of sensor networks, the sensor nodes that are far away from the base station have more difficulty getting 
bandwidth than sensor nodes near the base station. The congestion control protocol should provide fair bandwidth allocation among all source nodes in WSNs.

One of the most common definitions of fairness is max-min fairness [38]. A flow rate $x$ is defined to be max-min fair if any rate $x_{i}$ cannot be increased without decreasing the other rate $x_{j}$ which is smaller than or equal to $x_{i}$.

In some cases, sensor nodes might have a different importance or priority and they need different throughput. Therefore, weighted proportional fairness is required. In this thesis, we design a novel wighted fairness algorithm for multi-path routing WSNs. It guarantees that nodes with a high priority index will obtain more bandwidth than ones with low a priority index. 


\section{Chapter 3}

\section{JOINT PRIORITY ALGORITHM}

In previous chapters, we have demonstrated that congestion in WSNs must be controlled in an effective and proper manner. Current, existing approaches have some limitations. For example, some prior studies have considered multi-path routing, but these studies do not address the fairness problem. Other efforts considered fairness and weighted fairness issues, but they assume single-path routing, which oversimplifies the fairness problem. In order to deal with the general case when no specific restrictions are placed on routing protocols, we developed a novel algorithm called the Joint Priority Algorithm (JPA), which eliminates congestion and achieves weighted fairness in multi-path and multi-hop wireless sensor networks. This proposed algorithm can work with most existing routing protocol. 


\subsection{Joint Priority Algorithm Notations}

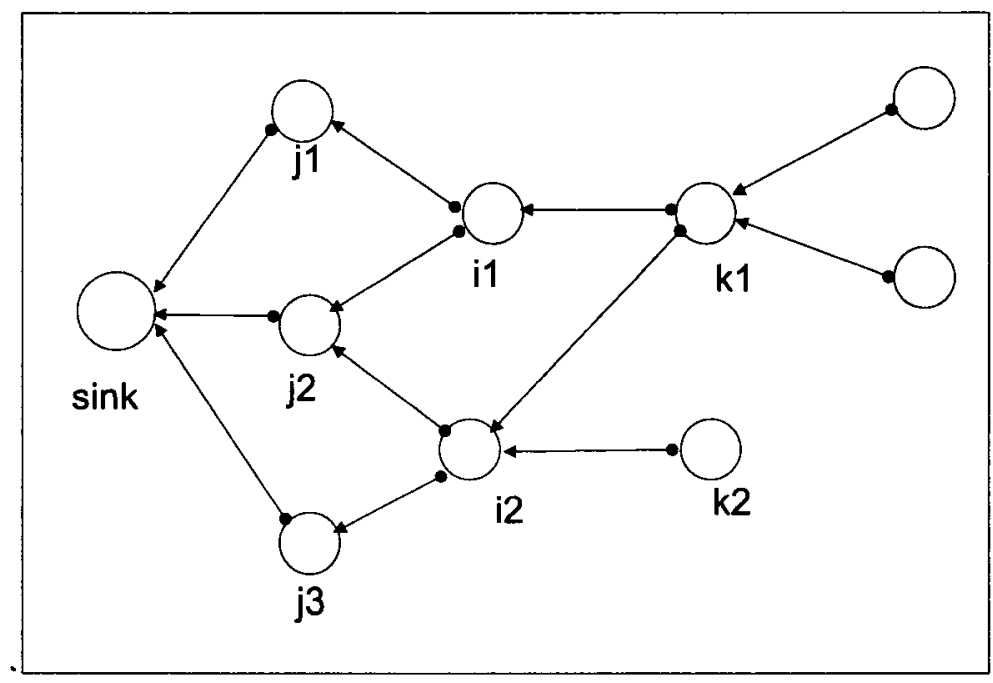

Figure 3.1: Network model in JPA

We studied one specific type of WSN where a set of base stations (sinks) are static and the location of the data source is fixed. In Figure 3.1, data packets are sent from data sources to base stations through a multi-path and multi-hop network and form convergent traffic in the upstream direction. Each data flow may pass through multiple paths before it arrives at the base station. The CSMA/CA MAC protocol is implemented in the MAC layer. Each sensor node could have two types of traffic: source traffic and transit traffic [7]. Source traffic is generated by the source nodes themselves, while transit traffic is relayed from other upstream nodes. Both of these are scheduled to the interface queue and form the outgoing traffic of that node. 


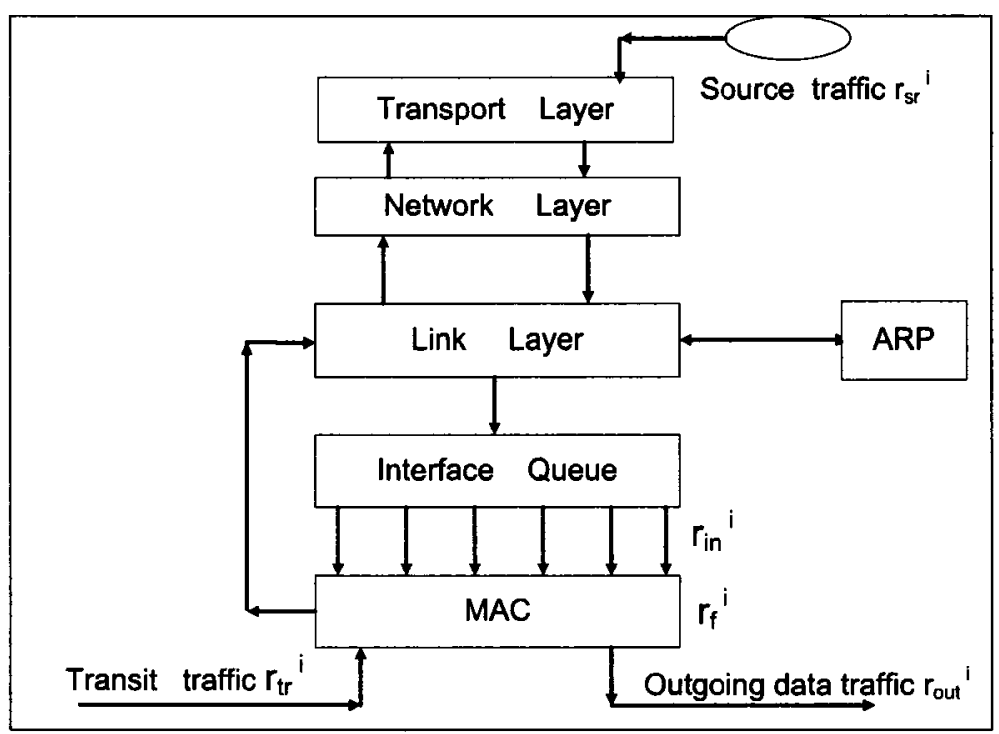

Figure 3.2: Node model in JPA

The node model of a particular sensor node is presented in Figure 3.2. The source traffic (ST) produced by this node is delivered to link layers through a network laycr. In the link layer, the transit traffic (TT) from its upstream neighbors and the source traffic are scheduled to the interface queue by the ARP (Address Resolution Protocol) protocol. Then, the packets are delivered to different downstream neighbors according to correct interfaces. As mentioned in the introduction, we employed the congestion detection mechanism presented in [8] to detect congestion. The main focus of this thesis is on the performance of the rate adjustment mechanism in response to congestion. We studied how upstream sensor nodes determine the right amount of rate deduction when downstream nodes are congested.

Before describing our algorithm, we need present some notations that will help 
describe it clearly. Let $N$ be the set of sensor nodes in a WSN; $D_{i}$ be the set of downstream neighbors of node $i$ and $U_{i}$ be the set of upstream neighbors of node $i$. $D_{i}$ and $U_{i}$ are determined by a routing protocol. $\forall j \in D_{i}$ and $\forall k \in U_{i},(i, j)$ are called downstream links of node $i$, while $(k, i)$ are called upstream links of node $i$. For example, in Figure 3.1, node $i_{2}$ has two upstream links: link $\left(k_{1}, i_{2}\right),\left(k_{2}, i_{2}\right)$ and two downstream links: link $\left(i_{2}, j_{2}\right),\left(i_{2}, j_{3}\right) . S T$ is defined as the source traffic of a node or link, while $T T$ is the transit traffic of a node or link. The sum of the source traffics and the transit traffics are globe traffic $(G T)$. So if $\forall i \in N$, we have following equation:

$$
\sum_{j \in D_{i}} G T(i, j)=\sum_{k \in U_{i}} G T(k, i)+S T(i)
$$

Equation (3.1) demonstrates that outgoing traffic of a particular node $i$ is equal to incoming traffic of that node plus its source traffic. This confirms the flow conversation theory.

Let $f$ be the flow generated by the data source node $f$ in network $N$. The data rate of flow $f$ is denoted as $r(f)$, while $r_{i}(f), r_{i, j}(f)$ and $r_{k, i}(f)$ are the rate at which the packets of flow $f$ pass through node $i$, downstream link $(i, j)$ and upstream link $(k, i)$ respectively. Obviously,

$$
r_{f}(f)=r(f)
$$

$r_{f}(f)$ is a special case of rate $r_{i}(f)$ at which the packets of flow $f$ pass through node $f$ itself. 
Eq. (3.1) results in the following equation: $\forall i$ and $f \in N$

$$
\begin{gathered}
r_{i}(f)=\sum_{k \in U_{i}} r_{k, i}(f), i \neq f \\
r_{i}(f)=\sum_{j \in D_{i}} r_{i, j}(f)
\end{gathered}
$$

Eq. (3.3) and Eq. (3.4) demonstrate that the rate at which the packets of flow $f$ pass through node $i$ is equal to the sum of data rates at which packets of flow $f$ pass through downstream link $(i, j)$ and also equal to the sum of data rates at which packets of flow $f$ pass through the upstream link $(k, i)$.

\section{$3.2 \quad$ Network Model}

In our work we addressed an upstream congestion control algorithm for WSNs that supports multi-path routing protocols. In Figure 3.1, sensor nodes generate continuous data and form many-to-one convergent traffic in the downstream direction. Data packets are delivered to the base station over multiple hops. Each sensor node can be a source node or an intermediate node. When a sensor node has upstream neighbors and transits the packets of its upstream neighbors, it is a source node as well as an intermediate node. On the other hand, it is only a source node if it has no upstream neighbors and therefore only has source traffic. For this multi-path network model, each intermediate node may not only have multiple upstream neighbors but will also 
have multiple downstream neighbors, which is different from the single-path network model (Figure 1.6). In the single-path network model, each intermediate node may have multiple upstream neighbors but only have one downstream neighbor. In our multi-path network model, this mean that the packets of a flow may be routed on many paths and only one fraction of a flow passes through a particular node or link. It is difficult to calculate the sum of the fractions of all data flows which pass through that node or link using the previous congestion control algorithm. For example, the priority-based congestion control protocol (PCCP)[7].

As an instance in Figure 3.1, the source node $k_{2}$ generates data flow $k_{2}$, and this flow will be relayed by the intermediate node $i_{2}$. However, the node $i_{2}$ has two downstream neighbors. That means flow $k 2$ will be delivered to the base station through two different links, $\left(i_{2}, j_{2}\right)$ and $\left(i_{2}, j_{3}\right)$. According to flow conversation theory, we have the following equation:

$$
r_{k_{2}}\left(k_{2}\right)=r_{i_{2}, j_{2}}\left(k_{2}\right)+r_{i_{2}, j_{3}}\left(k_{2}\right)
$$

Bsed on this equation, we notice that only part of flow $k_{2}$ passes through link $\left(i_{2}, j_{2}\right)$. Under this situation, if congestion occurs in node $j_{2}$, node $j_{2}$ cannot simply determine the amount of rate deduction in node $k_{2}$ because only part of data flow $k_{2}$ contributes to the congestion of node $j_{2}$.

Another problem we will address is end-to-end fairness and end-to-end weighted 
fairness issues in such a network model. The ideal congestion control approach should guarantee:

1. If no congestion occurs, source nodes send data packets at their preset sending rates.

2. In response to congestion, the source nodes with the same source priority send packets at almost the same rate, which confirms end-to-end fairness.

3. When congestion occurs, the source node with high priority achieve a higher sending rate than the one with a lower source priority, which indicates end-toend weighted fairness.

Each sensor node in such a network will reset its sending rate in response to congestion. A rate adjustment algorithm determines the right amount of rate deduction in order to guarantee ideal results.

\subsection{Node Model}

Figure 3.2 presents the node model of the JPA at the particular sensor node $i$. The incoming traffic includes the source traffic generated by itself and transit traffic coming from its upstream neighbors. All incoming traffic is scheduled to the interface queue according to the ARP (Address Resolution Protocol) table and form the outgoing 
traffic of that particular node. The data rate of source traffic is denoted as $r_{s r}^{i}$ and the data rate of transit traffic is denoted as $r_{t r}^{i} . r_{i n}^{i}$ and $r_{o u t}^{i}$ are the incoming and outgoing data rates of the particular interface of node $i$, while $r_{f}^{i}$ is the forwarding rate. Packets could be queued at the MAC layer if the incoming traffic rate $r_{i n}^{i}$ exceeds the packet forwarding rate $r_{f}^{i}$. The packet forwarding rate $r_{f}^{i}$ depends on the MAC protocol itself. In our work, the CSMA/CA MAC protocol was implemented. Therefore, the number of active sensor nodes and their traffic density will influence $r_{f}^{i}$. If $r_{i n}^{i}$ is smaller than $r_{f}^{i}, r_{\text {out }}^{i}$ will be equal to $r_{i n}^{i}$. Otherwise, $r_{\text {out }}^{i}$ will be close to $r_{f}^{i}$

Radio collision and buffer overflow are the two main forms of congestion in WSNs. The first one is called link-level congestion and the second one is called node-level congestion. Node-level congestion will take place when packets incoming rate exceeds the packet forwarding rate. In this case, we need to reduce the $r_{i n}^{i}$ through throttling either $r_{s r}^{i}$ or $r_{t r}^{i}$. The source rate $r_{s r}^{i}$ can be reduced locally by changing sampling frequency. The transit rate $r_{t r}^{i}$ can be reduced through rate adjustment at its upstream neighbors. On the other hand, link-level congestion occurs if there is collision on the link around the node $i$. When it occurs, the node $i$ and its neighbors should reduce channel access in order to prevent further link-level congestion.

In our work, we studies node-level congestion and designed a novel rate adjustment algorithm in each sensor node as shown in Figure 3.2. The weighted fair queue is 


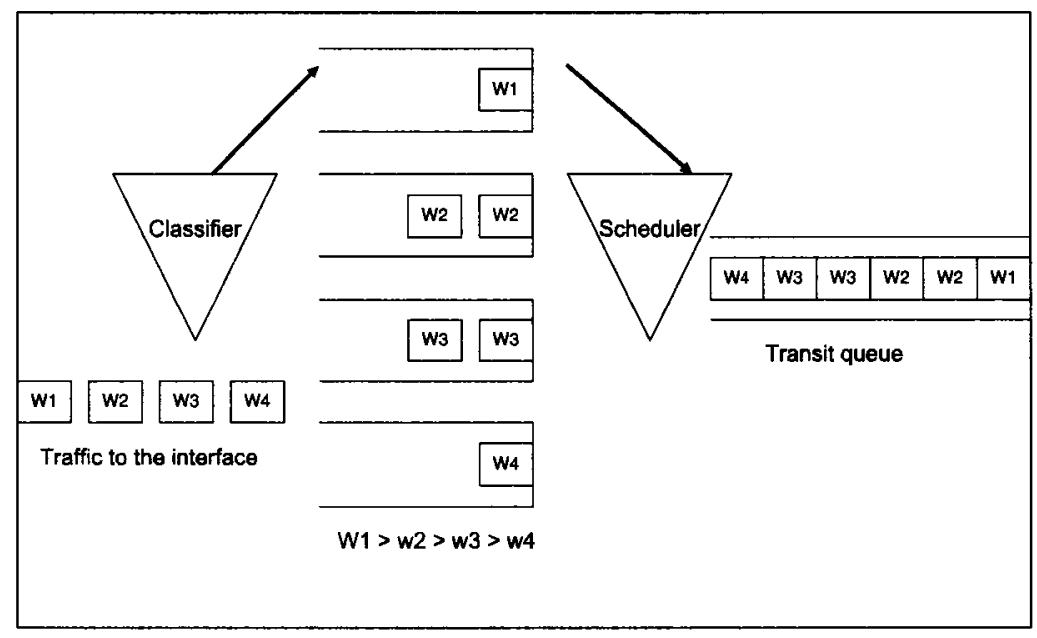

Figure 3.3: Weighted fair queue model

employed in the interface to guarantee the fairness between different data flows. See Figure 3.3 [39]. In this Figure, $w 1, w 2, w 3$ and $w 4$ are denoted as the queue weights. The flow with the high weight will be scheduled to transit the queue earlier than the flow with the low weight. Meanwhile, the priority index of each source node is used as the weight for each queue, which indicates the importance of each flow.

\subsection{The Joint Priority Algorithm}

The JPA was designed because in WSNs, congestion control protocols should guarantee end-to-cnd fairness for different sensor nodes so that each sensor nodes can achieve fair throughput. Otherwise, the biased sensor node cannot report events in their area 
and the system may misreport that there is no event in the area. Moreover, since the sensor node might have a different priority due to its function or location, congestion control protocols need to guarantee end-to-end weighted fairness so that the base station can get different throughput from sensor nodes in a weighted fair manner. The JPA was developed to avoid or reduce packet loss while guaranteeing end-to-end weighted fairness with lower control overhead in multi-path routing WSNs.

\subsubsection{Challenges of end-to-end fairness and end-to-end weighted fairness}

Many previous studies have examined MAC-layer fairness among one-hop flows within a neighborhood[40][38][41]. Other related works studying fairness at the MAC layer include [42] and [43]. In our work, we study network-layer fairness among end-to-end flows. The packets of a flow may be routed along different paths to a base station. When all routing paths from the data source to the sink are congested, the source has to reduce the rate at which it produces packets because the excess packets will be dropped anyway.

Congestion and fairness are two related but different problems. Resolving congestion does not guarantee fairness. For example, in Figure 3.4, the node 1, 2 and 3 are data sources and node 4 and 5 are relay nodes. The congestion control mechanism proposed in [44] limits the forwarding rate of sensor node 5 to four packets per sec- 


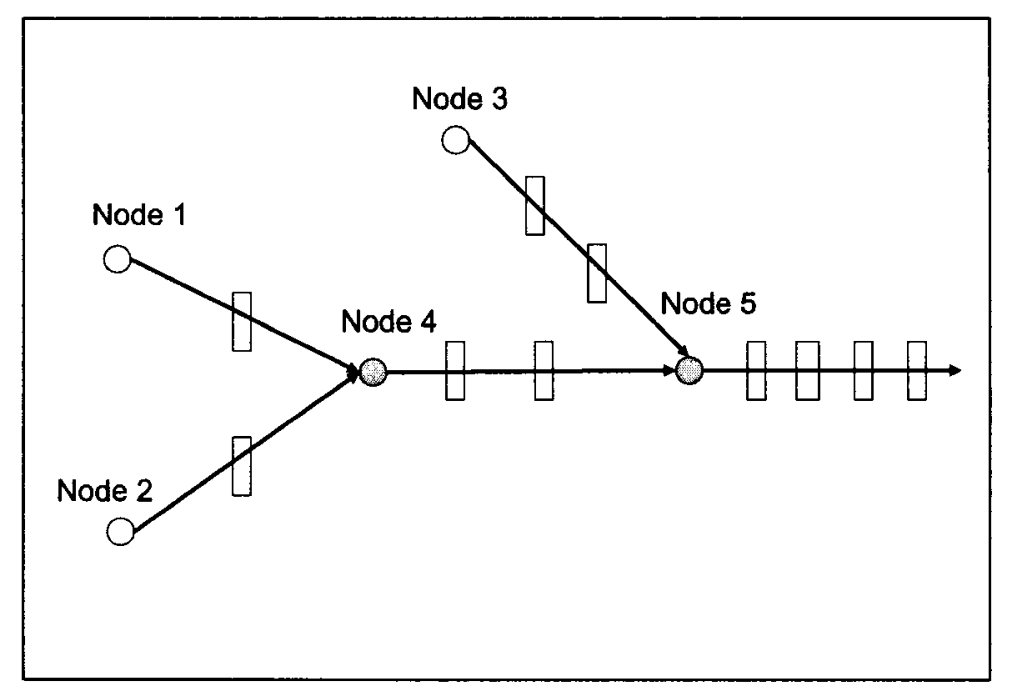

Figure 3.4: A topology of local fairness example

ond in order to avoid downstream congestion. The combined incoming rate to node 5 should be equal to the forwarding rate of node 5 over a long time. Therefore, node 3 and 4 which are the upstream neighbors of node 5 , can each send node 5 two packets per second. The further upstream neighbors, node 1 and 2 can only send one packet per second on average. Consequently, data source node 3 is able to generate packets at two times the average rate of node 1 and node 2 . The purpose of fairness is to distribute the available bandwidth between all the data sources, so that each upstream data source receives a fair or weighted fair share. Obviously, it is insufficient that the combined rate of node 3 and 4 does not exceed the forwarding rate of node 5 in [44].

A real sensor network has a far more complex topology than the one in Figure 3.4. A flow may follow numerous forwarding paths to reach the base station. In multi- 
path routing, the paths of all flows may form a complex DAG (directed acyclic graph) instead of a routing tree. The geographic routing [45] [46][47]creats a DAG, where a progress threshold may be used to select a candidate for the next routing hop. Achieving end-to-end fairness and end-to-end weighted fairness in a multi-path routing network is harder than in a tree-base routing network. At a forwarding node, the bandwidth should not be evenly divided among the passing flows because some flows may receive another bandwidth from other paths while other flows may not. The flows recciving other bandwidth from other paths should obtain less bandwidth than others that receive nothing from other paths.

\subsubsection{The proposed algorithm}

According to the theory presented in previous sections, in order to achieve fairness or weighted fairness in multi-path routing networks, it is insufficient to evenly divide the bandwidth among passing flows. We propose a Joint Priority-based Algorithm (JPA) that eliminates congestion and achieves weighted fairness in multi-path and multi-hop wireless sensor networks. The basic idea of the JPA is to allocate the bandwidth of each upstream link proportionally to the mean number of flows which flow to this congested node through that upstream link. For example, in Figure 3.4, when congestion occurs in node 5 , it estimates that one flow comes through a link from node 3 to node 5 , while two flows come from node 4 to node 5 . Based on 
the theory of JPA, node 5 will enforce the link rate from node 3 to node 5 to $\frac{1}{3} \times$ (forwarding rate of node 5), while the link rate from node 4 to node 5 is limited to $\frac{2}{3} \times($ forwarding rate of node 5$)$. It is difficult to maintain the per-flow information of a particular node in multi-path routing WSNs because the packets of a flow may be routed on many different paths.

The problem we studied in this thesis was how to estimate the mean number of flows which flow to this congested node through the upstream link in multi-path WSNs. If the intermediate nodes know the mean number and allocate the bandwidth proportionally to it, end-to-end weighted fairness will be guaranteed in multi-path routing WSNs.

According to the JPA, each source node has a source priority $(S P)$ which indicates the importance of the data flow generated by that source node. A new variable, joint priority $(J P)$ of nodes or links is introduced in this thesis. It is defined as the expected value of the random variable SP of the source node. The JP of a node or link indicates the arithmetic means of SP of the source nodes whose data flows pass through that particular node or link. Obviously, if we allocate bandwidth proportionally to $J P$, end-to-end weighted fairness will be guaranteed.

The probability at which data flow $f$ passes through node $i$ is denoted as $p_{i}(f)$ and the probability at which data flow $f$ passes through link $(i, j)$ and link $(k, i)$ is denoted as $p_{i, j}(f)$ and $p_{k, i}(f)$ respectively. According to basic probability theory and 
the definition of joint priority, we can calculate the $p_{i}(f), p_{i, j}(f)$ and $p_{k, i}(f)$ as follows:

$$
\begin{gathered}
p_{i}(f)=\frac{r_{i}(f)}{r(f)} \\
p_{i, j}(f)=\frac{r_{i, j}(f)}{r(f)} \\
p_{k, i}(f)=\frac{r_{k, i}(f)}{r(f)}
\end{gathered}
$$

$\forall i \in N$, let $J P(i)$ be the joint priority of node $i$, then

$$
\begin{aligned}
J P(i) & =\sum_{f \in N} p_{i}(f) \times S P(f) \\
& =\sum_{f \in N} \frac{r_{i}(f)}{r(f)} \times S P(f)
\end{aligned}
$$

$r(f)$ is data rate of flow $f$. Let $J P(i, j)$ be the joint priority of downstream link of node $i$, then

$$
\begin{aligned}
J P(i, j) & =\sum_{f \in N} p_{i, j}(f) \times S P(f) \\
& =\sum_{f \in N} \frac{r_{i, j}(f)}{r(f)} \times S P(f)
\end{aligned}
$$


If $J P(k, i)$ is the joint priority of upstream link of node $i$, then

$$
\begin{aligned}
J P(k, i) & =\sum_{f \in N} p_{k, i}(f) \times S P(f) \\
& =\sum_{f \in N} \frac{r_{k, i}(f)}{r(f)} \times S P(f)
\end{aligned}
$$

Bsed on Eq. (3.9), Eq. (3.10) and Eq. (3.11), the joint priority of a node or link is related to the per-flow state of nodes and links. For example, the variable $r_{i}(f)$, $r_{i, j}(f)$ and $r_{k, i}(f)$ are the data rate which are related to the flow $f$. Because the intermediate nodes cannot keep the per-flow information, it is impossible to calculate these variables. However we can prove that the joint priority of a node or link can be achieved iteratively by measuring the throughput of a particular node or link. According to flow conservation theory and Eq. (3.3) and Eq. (3.4), the joint priority of intermediate node $i$ is computed as:

$$
\begin{aligned}
J P(i) & =\sum_{f \in N} \frac{r_{i}(f)}{r(f)} \times S P(f) \\
& =\sum_{f \in N, f \neq i} \frac{r_{i}(f)}{r(f)} \times S P(f)+S P(i) \\
& =\sum_{f \in N, f \neq i} \frac{\sum_{k \in U_{i}} r_{k, i}(f)}{r(f)} \times S P(f)+S P(i) \\
& =\sum_{k \in U_{i}} \sum_{f \in N, f \neq i} \frac{r_{k, i}(f)}{r(f)} \times S P(f)+S P(i) \\
& =\sum_{k \in U_{i}} J P(k, i)+S P(i)
\end{aligned}
$$


Based on Eq. (3.12), we are able to conclude that the joint priority of a intermediate node is equal to the sum of the joint priority of its upstream links and its source priority.

The joint priority of the downstream link $(i, j)$ of node $i$ can be calculated as:

$$
\begin{aligned}
J P(i, j) & =\sum_{f \in N} \frac{r_{i, j}(f)}{r(f)} \times S P(f) \\
& =\sum_{f \in N} \frac{r_{i}(f) \times \frac{r_{i, j}}{r_{i}}}{r(f)} \times S P(f) \\
& =\sum_{f \in N} \frac{r_{i}(f)}{r(f)} \times S P(f) \times \frac{r_{i, j}}{r_{i}} \\
& =J P(i) \times \frac{r_{i, j}}{r_{i}}
\end{aligned}
$$

Where the $r_{i}$ and $r_{i, j}$ are the throughput of node $i$ and link $(i, j)$. They can be measured locally. From Eq. (3.13), we can see that the joint priority of downstream link $(i, j)$ is proportional to the data rate $r_{i, j}$ passing that link. Named as $J P(i, j) \propto r_{i, j}, j \in D_{i}$.

Now we can separate nodes to different layers along with the data flow direction from source nodes to base stations. The furthest source nodes are considered as the first layer at which the sensor nodes do not have upstream neighbors. The maximum number of layers is bounded by the length of the longest routing path. The nodes in the first layers will determine their joint priorities after the first iteration by Eq. (3.12). The joint priority of their downstream links will be calculated by Eq. (3.13), which is 
also the joint priority of the upstream links of sensor nodes in the second layers. After calculating the $J P$ of the second layer nodes by Eq. (3.12), the $J P$ of downstream links of sensor nodes in the second layers can again be solved by Eq. (3.13). By following the same process, the $J P$ of nodes and links in the whole WSN will be achieved after certain iterations.

For instance, in Figure 3.1, $J P\left(k_{2}\right)$ will be computed after this first iteration. The $J P$ of downstream links $\left(k_{2}, i_{2}\right)$ will be computed by Eq. (3.13). Then node $k_{2}$ sends the values of $J P\left(k_{2}, i_{2}\right)$ to its neighbor node $i_{2}$. The $J P$ of node $i_{2}$ will be calculated by $\mathrm{Eq} .(3.12)$ based on the values of $J P\left(k_{2}, i_{2}\right)$ and $J P\left(k_{1}, i_{2}\right)$. This process is repeated until the joint priorities of all nodes and links are known.

Each sensor node maintains these variables $(J P)$ which are updated periodically. In order to achieve end-to-end weighted fairness, sensor nodes should allocate the bandwidth of their upstream links proportionally to the value of $J P$. We employed the congestion detection approach from $[8]$ in which congestion is detected by monitoring the buffer space in the intermediate nodes. When sensor node $i$ is congested, it computes a rate limit $l(k, i)$ for each of its upstream neighbors $k$ as:

$$
l(k, i)=\frac{J P(k, i)}{J P(i)} \times r(i)
$$

In Eq. (3.14), the congested sensor nodes allocate the bandwidth of their upstream neighbors proportionally to the value of $J P(k, i) . r(i)$ is the current throughput of 
sensor node $i$ which can be measured locally. Node $i$ advertises $l(k, i)$ to its upstream neighbor $k$ by piggybacking in an ACK packet. After receiving the $l(k, i)$, sensor node $k$ uses the token-bucket algorithm to enforce the rate limit. Figure 3.5 shows how the token bucket algorithm works.

In the token bucket flowchart, $c$ is denoted as a counter of the token, and $t$ is a time variable. The actual rate of an upstream link will be bounded by the token generation rate $r$. When we change the $r$ to an expected limited rate $l(k, i)$, then the rate of its upstream neighbors will be regulated to $l(k, i)$. Sensor nodes which are data sources limit the data generation rate using a token bucket as follows:

$$
l(i)=\frac{S P(i)}{J P(i)} \times r(i)
$$

After an upstream neighbor enforces a rate limit, it may become congested because its sending rate is reduced. If its buffer is full, it will enforce rate limits on its upstream neighbors in a similar way. This process will repeat toward the data source. Finally, all affected data sources will adjust their sending rate to the fair bandwidth. These rate limits reflect the network load on the congested paths.

After the sending rate of upstream links and nodes are enforced to limited rates, both the source traffic and transit traffic are controlled in a priority-based weighted fair way. This fact is confirmed by our simulation results in the next chapter. 


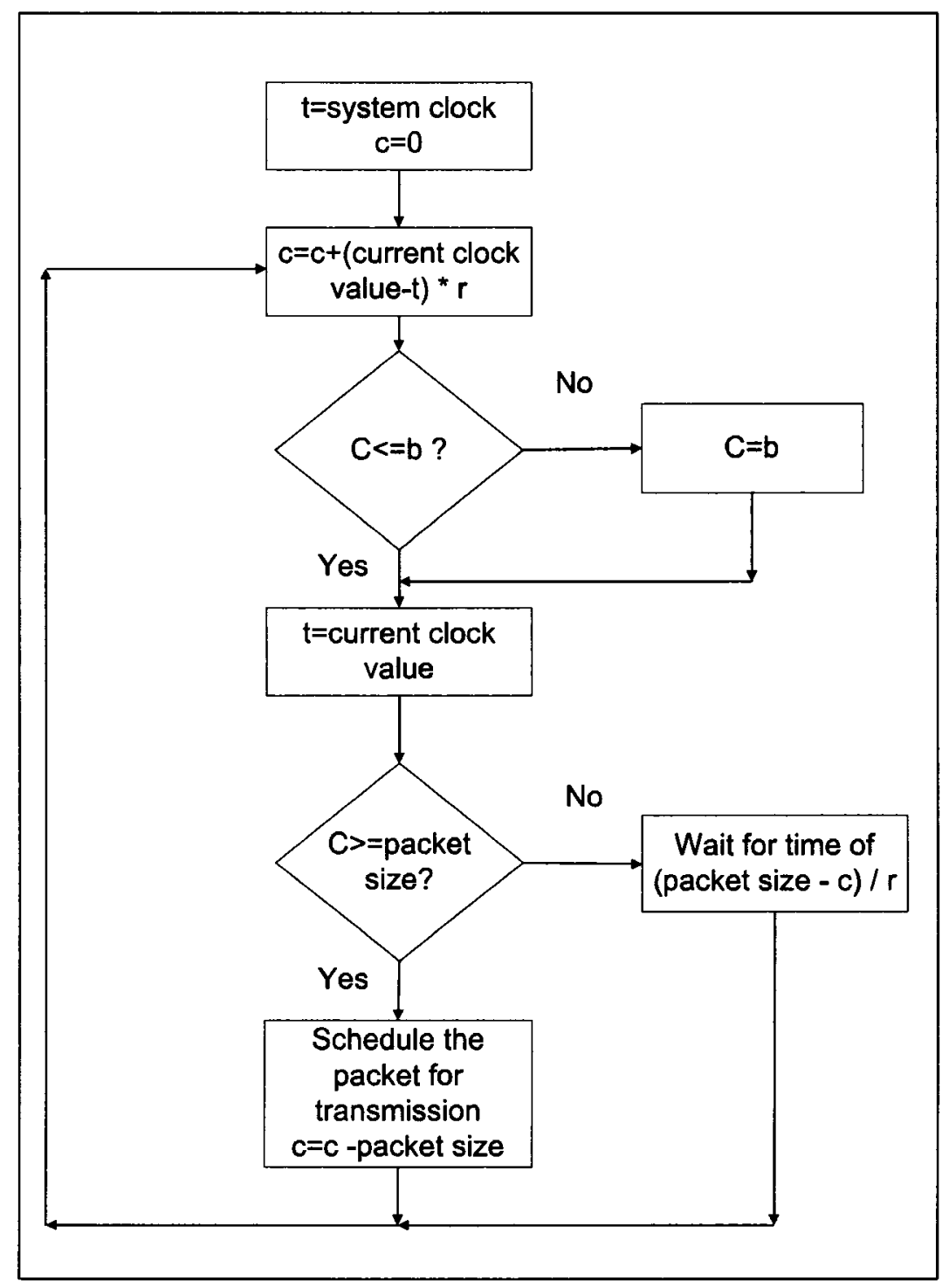

Figure 3.5: Flowchart of token bucket algorithm 


\section{Chapter 4}

\section{SIMULATION ENVIRONMENT}

\section{AND RESULTS ANALYSIS}

This chapter presents the results of the JPA simulation study. The first section will disscus the simulation environment. Next, the simulation results analysis will be presented. The analysis will address and compare the fairness results of three congestion control protocols: Congestion detection and avoidance (CODA) [9], Priority based congestion control protocol (PCCP) [7] and the proposed Joint Priority Algorithm. Finally, by presenting the simulation results we will demonstrate how the JPA guarantees end-to-end weighted fairness. 


\subsection{Simulation Environment}

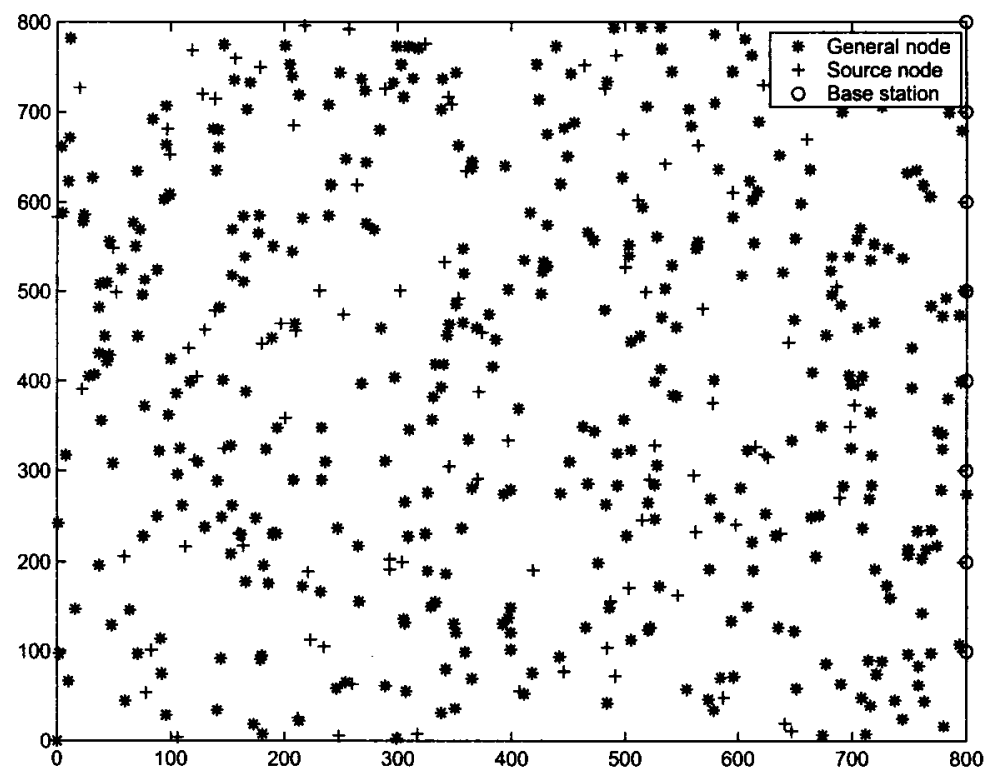

Figure 4.1: Network simulation topology

We simulated the proposed algorithm using $\mathrm{C}++$, and analyzed the simulation results using MATLAB. The simulation environment contained 8 base stations that were uniformly placed in the left edge of an $800 \times 800$ area. 400 sensor nodes and 80 data sources were randomly distributed in this area (See Figure 4.1). The transmission range of each sensor node was 80 . The neighbors of a sensor node were determined by the distance between this node and the others: if the distance between the two nodes was less than the transmission range, the two nodes were considered to be neighbors. Otherwise, they were not considered neighbors. Each source was assumed to produce data packets at a constant rate. In order to evaluate the performance of the proposed algorithm, we performed the simulation at 20 packets per time unit and 
25 packets per time unit. The size of data packets and control packets was 30 and 3 bytes respectively. Simulation parameters are listed in Table. 4.1. The CSMA/CA MAC protocol is implemented in the MAC layer.

Table 4.1: Fairness simulation parameters

\begin{tabular}{|c|c|}
\hline Network coverage & $800 \times 800$ \\
\hline Number of nodes & 400 \\
\hline Number of sources & 80 \\
\hline Number of sinks & 8 \\
\hline Transmission range & 80 \\
\hline Sending rate & 20 and 25 \\
\hline Size of data packets & 30 \\
\hline Size of control packets & 3 \\
\hline Size of buffer & 30 \\
\hline Source priority of nodes & 1 \\
\hline
\end{tabular}

\subsection{Simulation Results Analysis}

\subsubsection{Fairness comparison}

Three congestion mechanisms were implemented in our simulation to compare endto-end fairness. We assumed that all data sources had the same source priority, which meant that the data source whose paths were congested should have achieved the same throughput. The CODA [9] and PCCP [7] are two representive congestion schemes that have been previously studied. We implemented these two schemes and compared end-to-end fairness with our approach. We also provided simulation results 
when no congestion control schemes were employed. CODA, JPA and no congestion control schemes used the multi-path routing. Each sensor had a set of downstream links. PCCP used the single-path routing. The data sending rate was considered as the performance metric of fairness comparison, which is the average number of packets that were successfully delivered from a data source to the base station during per time unit.

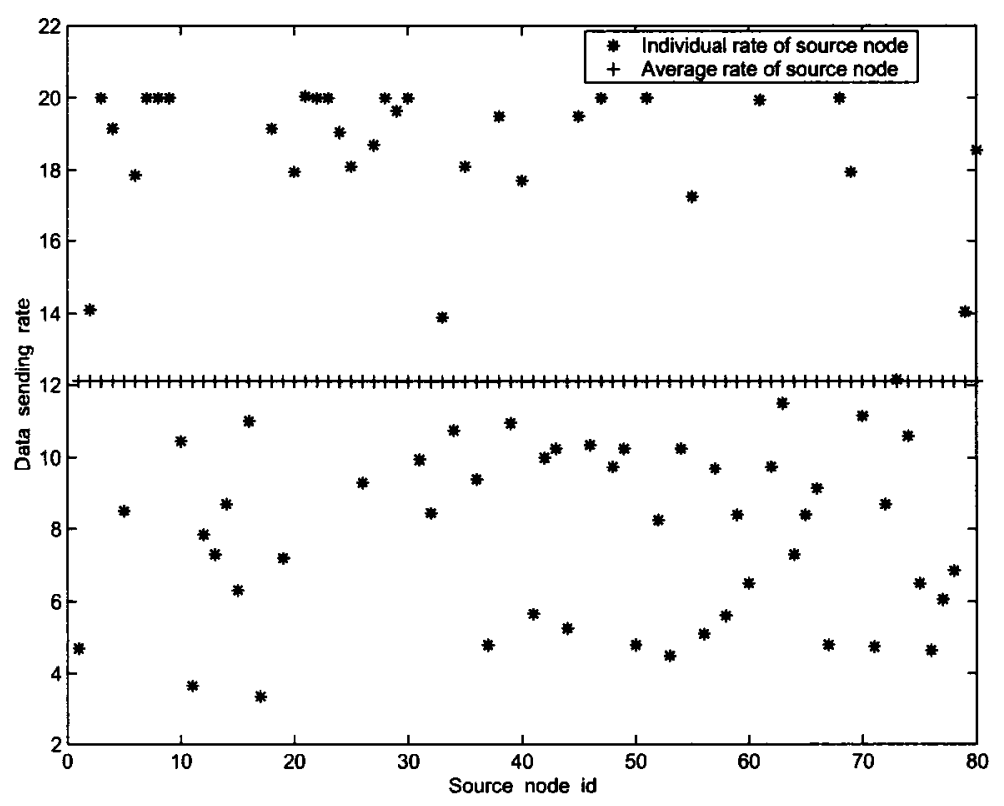

Figure 4.2: Simulation result of end-to-end fairness in no congestion control

When there were no congestion control mechanisms, source nodes and intermediate nodes do not adjust the sending rate. They were only controlled by the CSMA/CA MAC protocol. The individual sending rate of source nodes was widely distributed from 1 to 20 packets per time unit. The average throughput was low due to the leak of a congestion control mechanism. See Figure 4.2. 


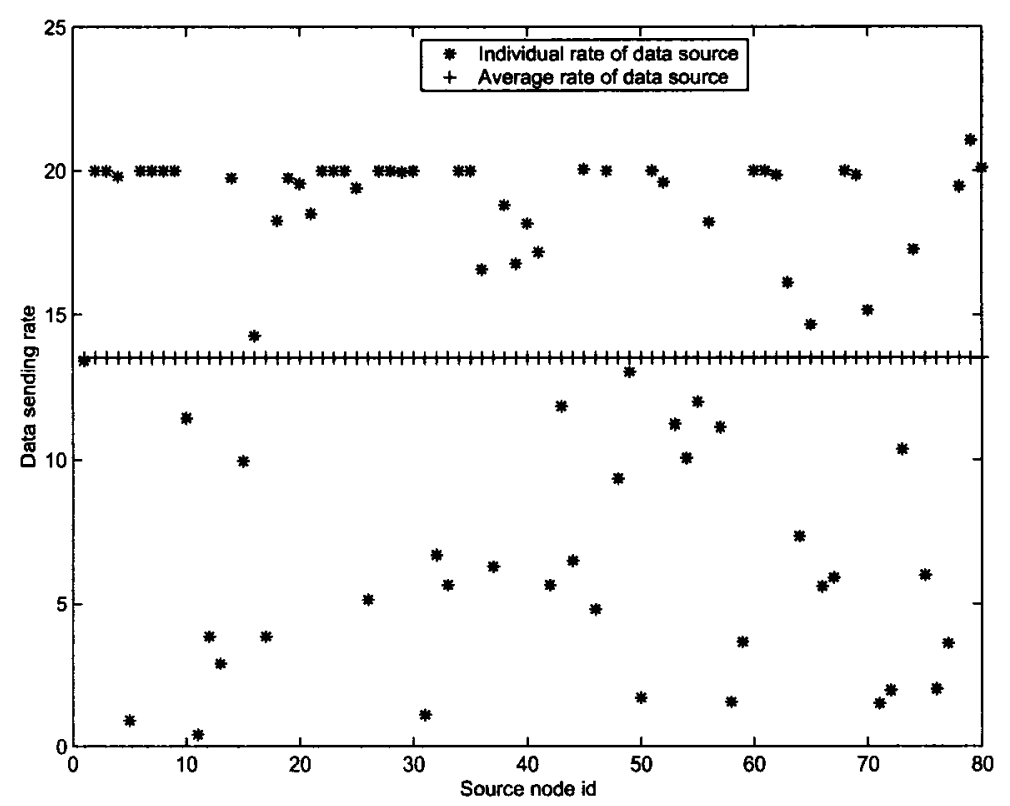

Figure 4.3: Simulation result of end-to-end fairness in CODA

CODA proposes an open-loop, hop-by-hop backpressure mechanism and a closedloop, multi-source regulation mechanism in event-driven WSNs. Sensor nodes detected congestion by monitoring the channel utilization and buffer-occupancy level. In response to congestion, the congested sensor node $i$ sent backpressure messages to its neighbors which may drop packets, reduce its forwarding rate to $i$ by $50 \%$ and further propagate backpressure messages. Figure 4.3 shows the simulation results. When no congestion occured, the data source sent the packets at a preset rate of 20 packets per time unit. In response to the congestion, the CODA only received the local fairness because the sending rate of all the upstream nodes was reduced at the same percentage even though the number of flows passing through those links was very different. The average rate of CODA is 13.5 packets per time unit. 


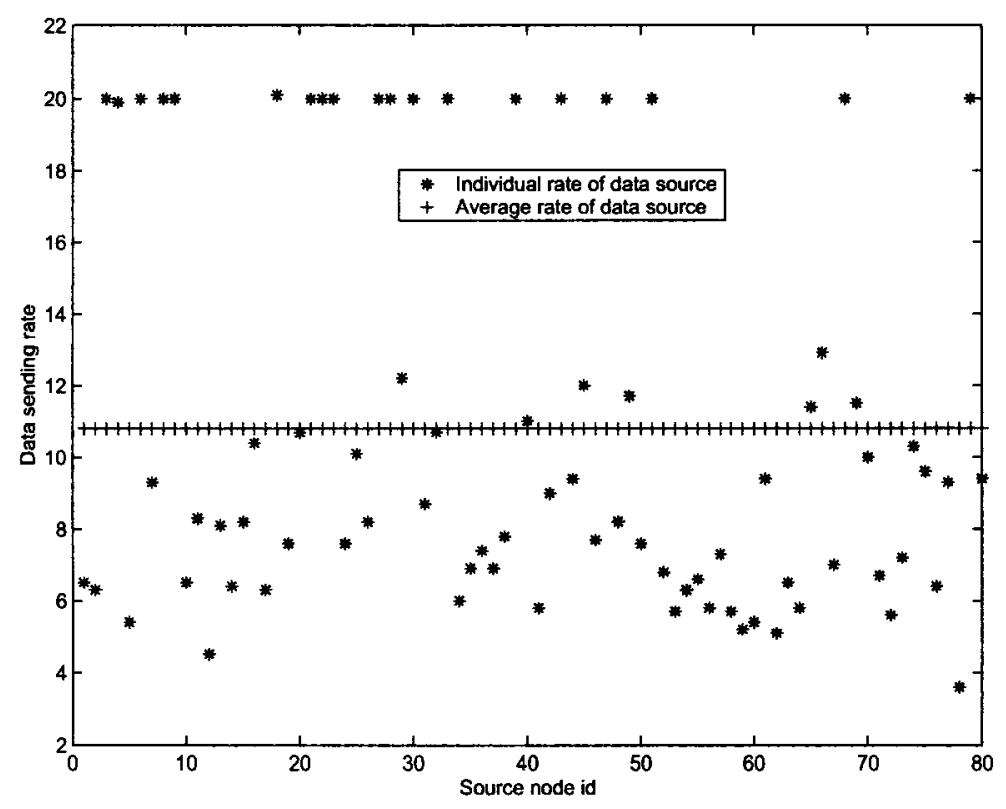

Figure 4.4: Simulation result of end-to-end fairness in PCCP

PCCP employs a hop-by-hop rate adjustment technique called priority-based rate adjustment (PRA) to adjust the scheduling rate and the source rate of each sensor node in single-path routing WSNs. Sensor nodes learn the number of upstream data sources in the subtree roots. When congestion occurs, the sensor node allocates the available bandwidth to upstream neighbors proportional to the number of upstream data sources. PCCP presented an end-to-end weighted fairness but assumes single-path routing only, which cannot work in multi-path routing WSNs. Figure 4.4 presents the simulation results. We can see that in response to congestion, the sending rates of data sources were regulated to a small range from 5 to 8 packets per time unit. The problem of PCCP was that the single-path routing can not fully exploit the capacity of the network. Under multi-path routing situation, PCCP was not able 
to work well. The average rate of PCCP was 10.8 packets per time unit.

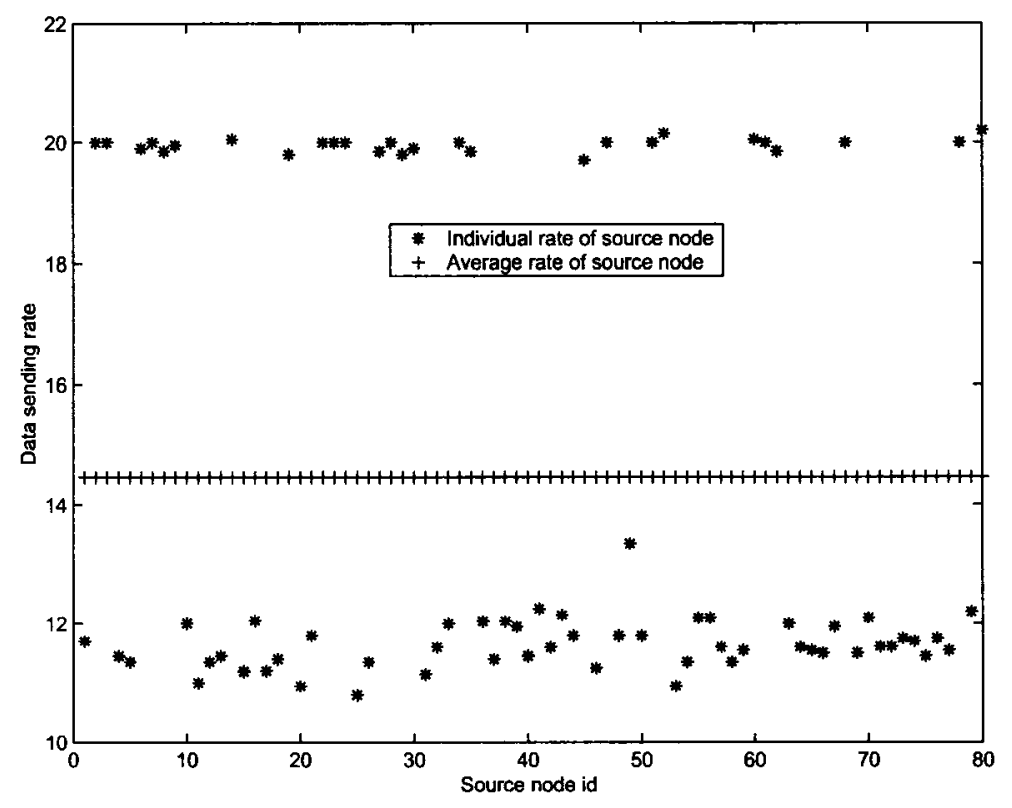

Figure 4.5: Simulation result of end-to-end fairness in JPA

In Figure 4.5, the simulation results demonstrate that the JPA improved fairness considerably. The sending rates of data sources fell into two groups. If the routing paths of a data source were not congested, the sending rate of this data source was a preset rate, 20 packets per time unit. Otherwise, the data source whose routing paths are congested were regulated to an almost equal sending rate in the range from 11 to 12 packets per time unit. This simulation result shows the JPA was able to achieve end-to-end fairness. Moreover, the average rate of the JPA is 14.46 packets per time unit, which is higher than the average rate of PCCP and CODA. 


\subsubsection{Achieving end-to-end weighted fairness}

Table 4.2: weighted fairness simulation parameters

\begin{tabular}{|l|c|c|c|c|}
\hline & Preset sending rate & \multicolumn{3}{|c|}{ SP of source nodes } \\
\cline { 2 - 5 } & (packets per time unit) & from 1 to 30 & from 31 to 60 & from 61 to 80 \\
\hline Simulation 1 & 20 & 3 & 2 & 1 \\
\hline Simulation 2 & 25 & 1 & 2 & 3 \\
\hline Simulation 3 & 25 & 3 & 1 & 2 \\
\hline Simulation 4 & 20 & 3 & 1 & 2 \\
\hline
\end{tabular}

It is possible that sensor nodes might be either outfitted with different sensors or geographically deployed in different places. Therefore, they may have different source priorities and need to gain different throughputs. In this situation, end-to-end weighted fairness is required so that sensor nodes can achieve a throughput proportional to their source priority. We present simulation results using four different scenarios and show that the JPA can guarantee end-to-end weighted fairness. The four simulation parameters are listed in Tab. 4.2

In the first simulation, we let the source priority of sensor nodes from number 1 to 30 be equal to 3 , equal to 2 from number 31 to 60 and equal to 1 for the last source nodes from number 61 to 80 . Data flow from source nodes passed through multiple paths with multiple hops before it arrived at the base station. The preset sending rate is 20 packets per time unit. Figure 4.6 shows simulation results. From these results it can be observed that: 


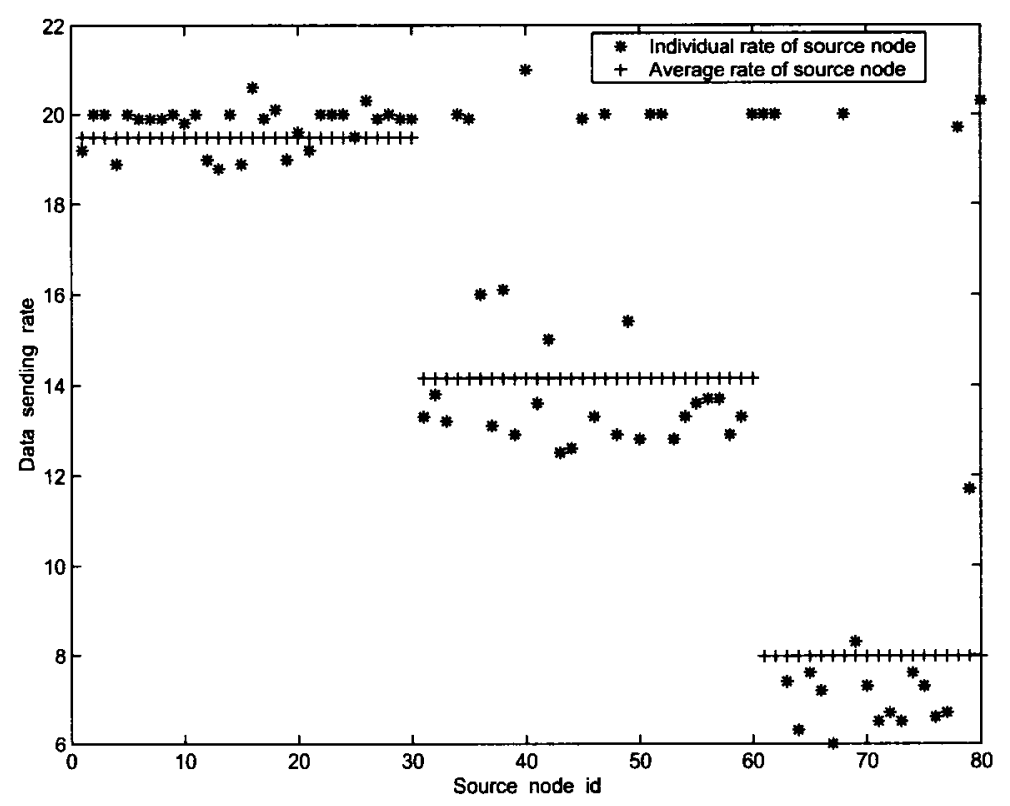

Figure 4.6: Simulation result 1 of end-to-end weighted fairness in JPA

1. If no congestion occurs in the routing paths, source nodes sent data packets at their preset sending rates which was 20 packets per time unit.

2. In response to congestion, the source nodes with the same source priority sent packets at almost the same rate, which confirms the fairness of congestion control. For example, source nodes from 1 to 30 who have the same source priority 3 limited their individual sending rate at around 14 data packets per unit time.

3. When congestion occured in the routing paths, the source node with high priority achieved a higher sending rate than the one with lower source priority, which indicates that the priority based weighted fairness of congestion control, in which sending rate of each source node is proportional to its source priority. 
From Figure4.6, we can see that the average rate of source nodes whose source priority is 3,2 and 1 are $19.48,14.15$ and 7.98 packets per time unit respectively. The average rates of nodes were roughly proportional to their source priority when their routing paths were congested.

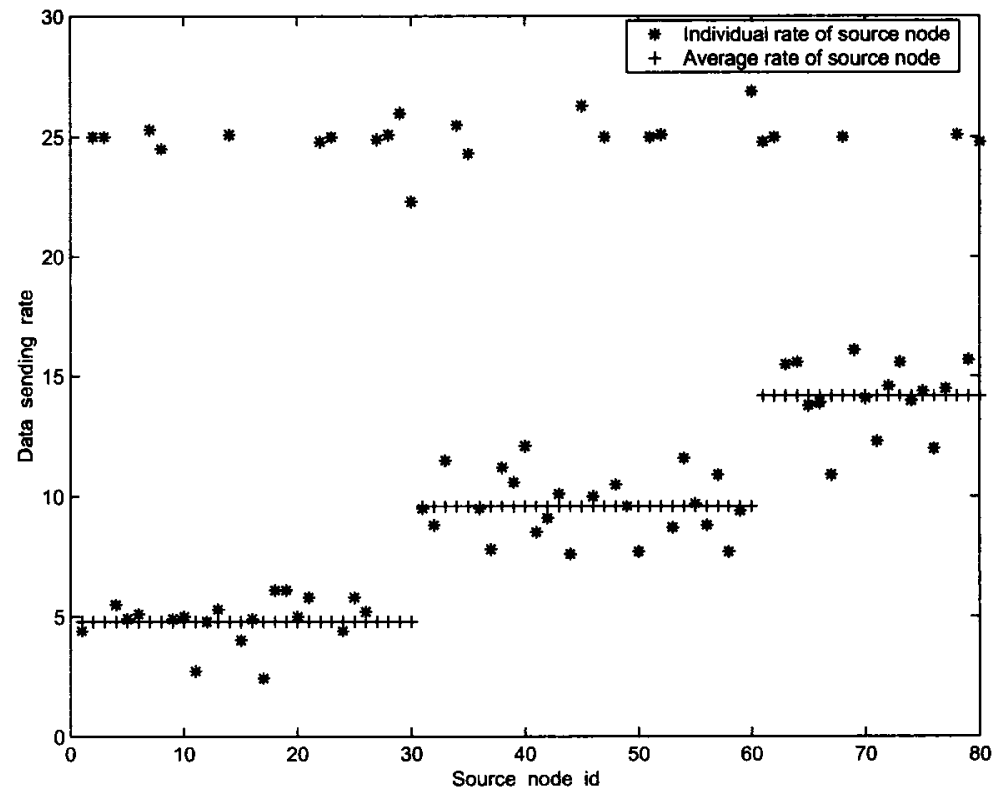

Figure 4.7: Simulation result 2 of end-to-end weighted fairness in JPA

In the second simulation, we let the source priority of sensor nodes from number 1 to 30 be equal to 1 , equal to 2 from number 31 to 60 and equal to 3 for the last source nodes from number 61 to 80 . The preset sending rate was 25 packets per time unit. Figure 4.7 shows the simulation results. We can see that the average rate of source nodes whose source priority is 3,2 and 1 were $4.8,9.6$ and 14.19 packets per time unit respectively. The average rate of nodes was roughly proportional to their source priority when their routing paths were congested. 


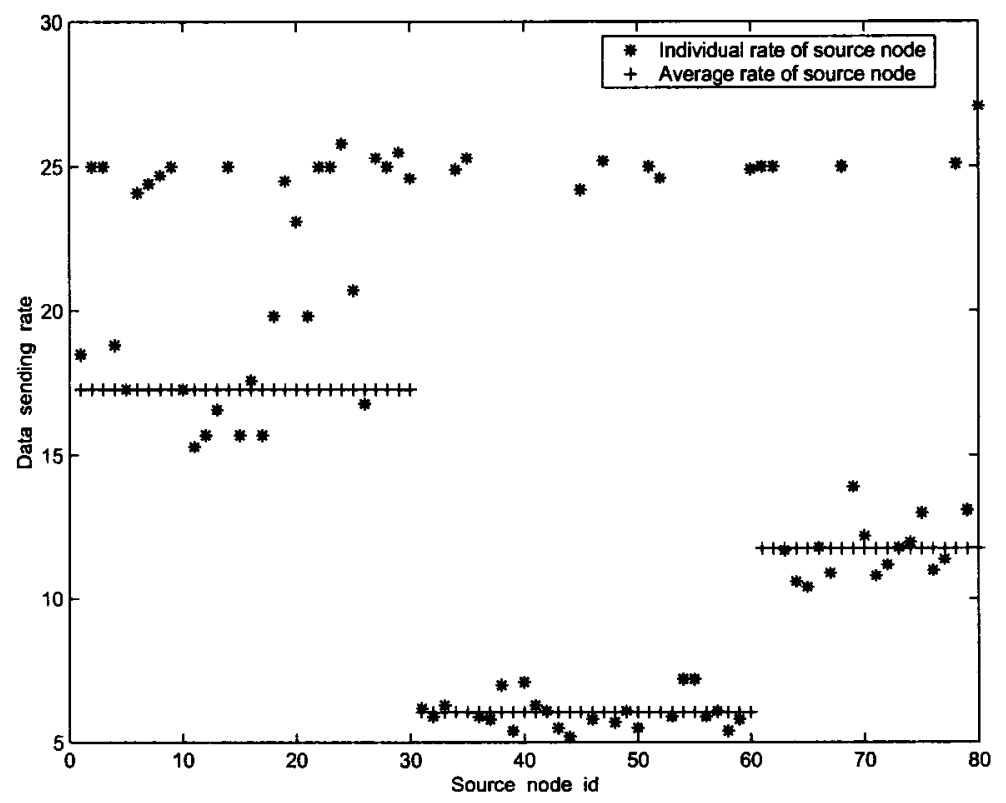

Figure 4.8: Simulation result 3 of end-to-end weighted fairness in JPA

In the third simulation, we let the source priority of sensor nodes from number 1 to 30 be equal to 3, equal to 1 from number 31 to 60 and equal to 2 for the last source nodes from number 61 to 80 . The preset sending rate was 25 packets per time unit. Figure 4.8 shows the simulation results. We can see that the average rate of source nodes whose source priority is 3,2 and 1 was $17.3,6.06$ and 11.77 packets per time unit respectively. The average rates of nodes were roughly proportional to their source priority when their routing paths were congested.

In the fourth simulation, we let the source priority of sensor nodes from number 1 to 30 be equal to 3 , equal to 1 from number 31 to 60 and equal to 2 for the last source nodes from number 61 to 80 . The preset sending rate was 20 packets per time 


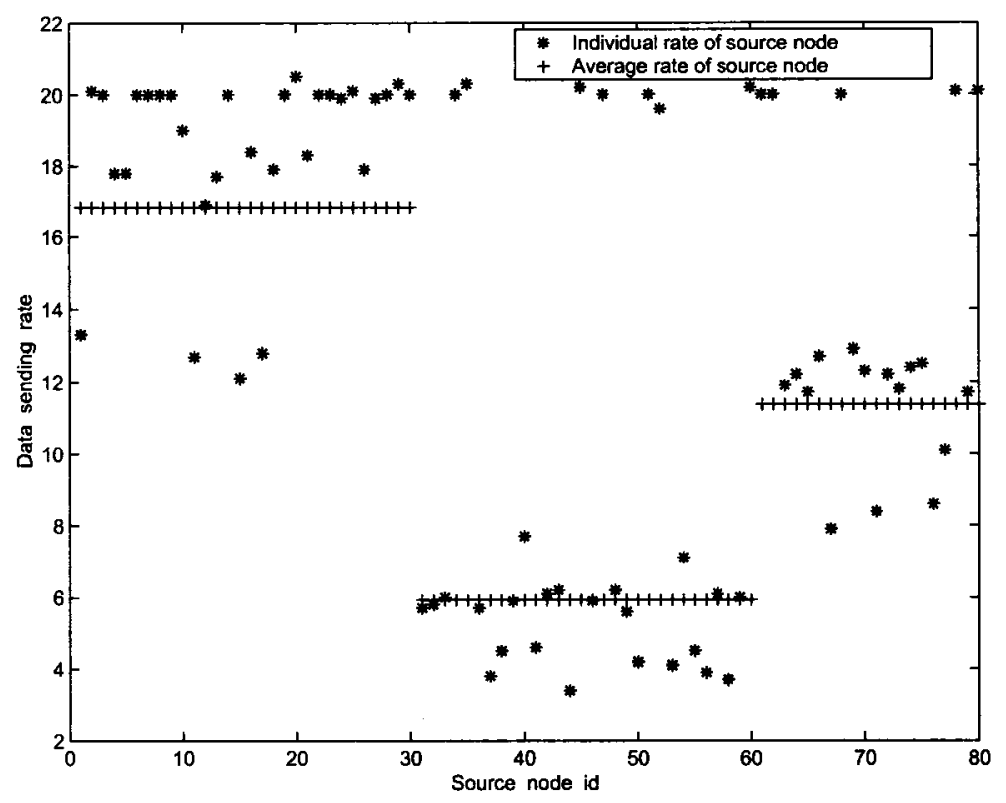

Figure 4.9: Simulation result 4 of end-to-end weighted fairness in JPA

unit. Figure 4.9 shows the simulation results. We can see that the average rate of source nodes whose source priority is 3,2 and 1 was $16.83,5.93$ and 11.37 packets per time unit respectively. The average rates of nodes were roughly proportional to their source priority when their routing paths were congested.

The same results can be observed from Figure4.7, Figure4.8 and Figure4.9. All results confirm that the JPA can achieve priority-based end-to-end fairness. 


\subsection{Summary of Simulation Results}

In this section, we presented simulation results and evaluated the performance of our proposed Joint Priority Algorithm. We also compared two previous studies with our approach. The advantages of the Joint Priority Algorithm were presented through a set of simulation results. In brief, the JPA performed better than the other two methods on end-to-end fairness. Furthermore, we demonstrated that the JPA achieves end-to-end weighted fairness. In response to congestion, the source nodes with high source priority will obtain the more bandwidth than the ones with low source priority. The proposed algorithm guarantees end-to-end fairness and end-to-end weighted fairness in multi-path networks, which considerable improves the current performance of congestion control protocols. 


\section{Chapter 5}

\section{CONCLUSIONS}

\subsection{Conclusions}

Wireless sensor networks have been used increasingly in recent years since they are low cost, easy to deploy and can work in any environment. However, wireless sensor networks are a newly emerging technology and there are many open issues about their communication protocols. In this thesis, we focused on the fairness and weighted fairness issues of congestion control protocol design. We believe that fairness and weighted fairness are one of the most important issues for the design of congestion control protocols in WSNs. In a WSN, each sensor node produces data packets, which should have an equal or weighted chance to access network resources.

This thesis began by reviewing current work on the congestion control protocols in 
wireless sensor networks. Then, we addressed the fairness issue of congestion control schemes. We noted that previous work on congestion control mechanisms did not guarantee end-to-end weighted fairness or end-to-end weighted fairness is ensured only in single-path routing WSNs. However, we believe that end-to-end weighted fairness is extremely important in the design of congestion control protocols because source nodes with high source priority should obtain more bandwidth than ones with low source priority in response to congestion. A new congestion control scheme, the Joint Priority Algorithm(JPA), was proposed for multi-path routing WSNs. JPA guarantees end-to-end fairness, as well as end-to-end weighted fairness and works on any routing protocol.

The new algorithm proposed in this thesis introduced a new variable called joint priority $(J P)$, which represents the expected value of random variable $S P$ of the source node. Each sensor node maintains these variables $(J P)$. The JPA allocates the bandwidth of each upstream link proportionally to the $J P$ of each node or link. As a result, source nodes with high source priority should obtain more bandwidth than ones with low source priority in response to congestion.

To evaluate the performance of this new algorithm, a simulation study was designed and implemented for different scenarios. The simulation results demonstrated that:

1. The proposed algorithm has the best performance on end-to-end fairness when 
compared with CODA and PCCP.

2. The new algorithm guarantees priority based end-to-end fairness. The sending rates of source nodes are roughly proportional to their source priority when their routing paths are congested.

For the new algorithm, we studied network-layer fairness among end-to-end flows. It was able to work at any routing protocol.

\section{$5.2 \quad$ Future Work}

Much of the work in this thesis has been concentrated on achieving fairness and weighted fairness in congestion control protocols for multi-path routing WSNs. We redeem that end-to-end fairness and end-to-end weighted fairness are one of the most important issues when congestion occurs in WSNs. However, a number of other issues still need to be considered in the future.

Firstly, it will be interesting to investigate energy-efficiency performance by comparing with other congestion control schemes, such as CODA and PCCP. In the future, we need to find an effective performance metric to evaluate the energy efficiency in our algorithm.

In our work, the number of dropped packets was not taken into account. This number should be low to save the limited power energy of WSNs. In the future, the 
accumulated number of dropped packets should to be calculated in our algorithm and compared with other congestion control schemes.

Finally, the impact of the number of hops between source nodes and base stations also deserves further investigation. In previous work, PCCP showed some results to evaluate this performance. In the case of multi-path routing WSNs, its impact will be more complicated. 


\section{Appendix A}

\section{Glossary of Terms}

ST - The source traffic of a node

TT - The transit traffic of a node

GT - The globe traffic of a node

SP - The source priority of of a node

JP - The joint priority of a node or link

$r(f)$ - The data rate of flow $f$

$r_{i}(f)$ - The rate at which the packets of flow $f$ pass through node $i$

$r_{i, j}(f)$ - The rate at which the packets of flow $f$ pass through downstream link $(i, j)$

$r_{k, i}(f)$ - The rate at which the packets of flow $f$ pass through upstream link $(k, i)$ 
$r_{s r}^{i}$ - The data rate of source traffic in node $i$

$r_{t r}^{i}$ - The data rate of transit traffic in node $i$

$r_{i n}^{i}$ - The incoming data rate of node $i$

$r_{\text {out }}^{i}$ - The outgoing data rate of node $i$

$r_{f}^{i}-$ The forwarding rate of node $i$

$p_{i}(f)$ - The probability at which data flow $f$ passes through node $i$

$p_{i, j}(f)$ - The probability at which data flow $f$ passes through link $(i, j)$

$p_{k, i}(f)$ - The probability at which data flow $f$ passes through link $(k, i)$

$l(k, i)$ - The limited rate of the upstream link $(k, i)$ when node $i$ is congested

$l(i)$ - The limited generation rate of data source $i$ when it is congested

$l a m b d a_{t+1}-$ The sending rate at time point $t+1$ in AIMD model

$l a m b d a_{t}-$ The sending rate at time point $t$ in AIMD model

$a_{I}-$ The parameter in AIMD model

$b_{D}-$ The parameter in AIMD model

$b_{D}-$ The parameter in AIMD model

$\lambda(t)$ - The source sending rate at time $t$ in rate-based AIMD model 
$q(t)$ - The bottleneck's queue size at time $t$ in rate-based AIMD model

$\mu$ - The bottleneck's service rate in rate-based AIMD model

$\tau_{F}$ - The propagation delay from the source to bottleneck in rate-based AIMD model

$\tau_{R}$ - The propagation delay from bottleneck to the source in rate-based AIMD model 


\section{Appendix B}

\section{Bibliography}




\section{Bibliography}

[1] I. Akyildiz, W. Su, Y. Sankarasubramaniam, and E.Cayirci, "Wireless sensor networks: A survey", in The Proceeding of Computer Networks, Vol. 38, pp. 393-422, 2002.

[2] C. Wang, K. Sohraby, and B. Li, "SenTCP: A hop-by-hop congestion control protocol for wireless sensor networks", in The Proceeding of IEEE INFOCOM 2005 (Poster Paper), Miami, Florida, USA, 2005.

[3] P. Agrawal, S. T. Tan, and A. L. Ananda, "A lightweight protocol for wireless sensor networks", in IEEE Wireless Communications and Networking 2003, Vol. 2, pp. 1280-1285, 2003.

[4] G.J.Pottie and W.J.Kaiser, "Wireless integrated network sensors", in Aerospace Conference Proceedings, Vol. 43, pp. 52-58, ACM Press, 2000.

[5] T. Gao, D. Greenspan, M. Welsh, R. R. Juang, and A. Alm, "Vital signs monitoring and patient tracking over a wireless network", in The Proceeding of 27th 
IEEE EMBS Annual International Conference, 2005.

[6] C. T. Ee and R. Bajcsy, "Congestion control and fairness for many-to-one routing in sensor networks", in The Proceeding of ACM Sensys'04, Baltimore, Maryland, USA, 2003.

[7] C. Wang, K. Sohraby, V. Lawrence, B. Li, and Y. Hu, "Priority-based congestion control in wireless sensor networks", in IEEE International Conference on Sensor Networks, Ubiquitous, and Trustworthy Computing (SUTC 2006), pp. 22-31, Taichung, Taiwan, 2006, IEEE Computer Society.

[8] S. Chen and N. Yang, "Congestion avoidance based on light-weight buffer management in sensor networks", in IEEE Transactions on Parallel and Distributed Systems, Special Issue on Localized Communication and Topology Protocols for Ad Hoc Networks, Vol. 17, 2006.

[9] C. Y. Wan, S. B. Eisenman, and A. T. Campbell, "CODA: Congestion detection and avoidance in sensor networks", in The Proceeding of ACM Sensys'03, Los Angeles, California, USA, 2003.

[10] B. Hull, K. Jamieson, and H. Balakrishnan, "Mitigating congestion in wireless sensor networks", in The Proceeding of ACM Sensys'04, Baltimore, Maryland,USA, 2004. 
[11] Y.G.Iyer, S.Gandham, and S.Venkatesan, "STCP: A generic transport layer protocol for wireless sensor networks", in The Proceeding of IEEE ICCCN, San Diego, California, USA, 2005.

[12] Y. S., O.B.Akan, and I. F.Akyildiz, "ESRT: Event-to-sink reliable transport in wireless sensor networks", in The Proceeding of MobiHoc03, Annapolis, Maryland, USA, 2003.

[13] D.-M. Chiu and R. Jain, "Analysis of the increase and decrease algorithms for congestion avoidance in computer networks", in Computer Networks, Vol. 17, pp. 1-14, 1989.

[14] J. Bolot and A. Shankar, "Dynamical behavior of rate-based flow control mechanisms", in ACM SIGCOMM Computer Communication Review, Vol. 20, pp. 35-49, 1990.

[15] P. Chimento, "Standard token bucket terminology", A Token Bucket Introduction.

[16] Al-Karaki, J.N.Kamal, and A.E, "Routing techniques in wireless sensor networks: a survey", in IEEE wireless communication, pp. 6-28, 2004.

[17] W.Heinzelman, J.Kulik, and H.Balakrishnan, "Adaptive protocols for information dissemination in wireless sensor networks", in The Proceeding of ACM/IEEE MobiCom'99, pp. 174-185, Seattle, USA, 1999. 
[18] C.Intanagonwiwat, R.Govindan, and D.Estrin, "Directed diffusion: a scalable and robust communication paradigm for sensor networks", in The Proceeding of ACM MobiCom'00, pp. 56-67, Boston, USA,, 2000.

[19] D.Braginsky and D.Estrin, "Rumor routing algorithm for sensor metworks", in The Proceeding of the first Workshopon Sensor Networks and Applications (WSNA), 2002.

[20] C.Schurgers and M.B.Srivastava, "Energy efficient routing in wireless sensor networks", in The Proceeding of the MILCON Proceedings on Communications for Network-Centric Operations: Creating the Information Force, 2001.

[21] S. L. L. Z. F. Ye, A. Chen, "A scalable solution to minimum cost forwarding in large sensor networks", in The Proceeding of the tenth International Conference on Computer Communications and Networks (ICCCN), 2001.

[22] W.Heinzelman, A.Chandrakasan, and H.Balakrishnan, "Energy efficient communication protocols for wireless microsensor networks", in The Proceeding of 33rd Hawaii International Conference on System Sciences, 2000.

[23] A. Manjeshwar and D. P. Agarwal, "TEEN: A routing protocol for enhanced eciency in wireless sensor networks", in 1st International Workshop on Parallel and Distributed Computing Issues in Wireless Networks and Mobile Computing, 2001. 
[24] A. Manjeshwar and D. P. Agarwal, "APTEEN: A hybrid protocol for ecient routing and comprehensive information retrieval in wireless sensor networks", in The Proceeding of International Parallel and Distributed Processing Symposium, 2002.

[25] L. Subramanian and R. H. Katz, "An architecture for building self congurable systems", in The Proceeding of IEEE/ACM Workshop on Mobile Ad Hoc Networking and Computing, 2000.

[26] C. R. S. Lindsey, "PEGASIS: Power-efficient gathering in sensor information systems", in The Proceeding of IEEE Aerospace Conference,, 2002.

[27] B.Chen, K.Jamieson, H.Dalakrishnan, and R.Morris, "SPAN: an energy-efficient coordination algorithm for topology maintenance in ad hoc wireless networks", in Wireless Networks, Vol. 8, pp. 481-494, 2002.

[28] D. E. Y. Yu and R. Govindan, "Geographical and energy-aware routing: A recursive data dissemination protocol for wireless sensor networks", in UCLA Computer Science Department Technical Report.

[29] I. Stojmenovic and X. Lin., "GEDIR: Loop-free location based routing in wireless networks", in International Conference on Parallel and Distributed Computing and Systems, Boston, MA, USA, 1999. 
[30] A. Z. F. Kuhn, R. Wattenhofer, "Worst-case optimal and average-case ecient geometric ad-hoc routing", in The Proceeding of the 4th ACM International Conference on Mobile Computing and Networking, 2003.

[31] S.Dulman, T.Nieberg, J.Wu, and P.Havinga, "Tade-off between traffic overhead and reliability in multipath routing for wireless sensor networks", in The Proceeding of WCNC Workshop, New Orleans, Louisiana, USA, 2003.

[32] S. S. D. Ganesan, R. Govindan and D. Estrin, "Highly-resilient, energy-efficiency multipath routing in wireless sensor networks", in ACM SIGMOBILE Mobile Computing and Communications Review, Vol. 5, 2001.

[33] Q. Li, J. Aslam, and D. Rus, "Hierarchical power-aware routing in sensor networks", in The Proceeding of the DIMACS Workshop on Pervasive Networking, 2001.

[34] J.-H. Chang and L. Tassiulas, "Maximum lifetime routing in wireless sensor networks", in The Proceeding of Advanced Telecommunications and Information Distribution Research Program (ATIRP2000), 2000.

[35] J. R. C. Rahul, "Energy aware routing for low energy ad hoc sensor networks", in IEEE Wireless Communications and Networking Conference (WCNC), Vol. 1, 2002. 
[36] D. E. N. Bulusu, J. Heidemann, "GPS-less low cost outdoor localization for very small devices", in Computer science department, University of Southern California, Technical report No. 00-729, 2000.

[37] J.Kulik, W.R.Heinzelman, and H.Balakrishnan, "Negotiation-based protocols for disseminating information in wireless sensor networks", in Wireless networks, Vol. 8, pp. 56-67, 2002.

[38] A. Sridharan and B. Krishnamachari, "Max-min fair collision-free scheduling for wireless sensor networks", in The Proceeding of Workshop on Multihop Wireless Networks, 2004.

[39] J.Mo and J.Walrand, "Fair end-to-end window-based congestion control", in IEEE/ACM Transactions on Networking, 2000.

[40] H. Luo, J. Cheng, and S. Lu, "Self-coordinating localized fair queueing in wireless ad hoc networks", in IEEE/ACM Transactions on Mobile Computing, Vol. 3, 2004.

[41] T. Nandagopal, T. Kim, X. Gao, and V. Bharghavan, "Achieving mac layer fairness in wireless packet networks", in The Proceeding of MobiCom'00, 2000.

[42] S. L. H. Luo and V. Bharghavan, "A new model for packet scheduling in multihop wireless networks", in The Proceeding of MobiCom'00, 2000. 
[43] Y. W. B. Bensaou and C. C. Ko, "Fair medium access in 802.11 based wireless ad-hoc networks", in The Proceeding of MobiHoc'00, Boston, Massachusetts, 2000.

[44] L.Tassiulas, "Adaptive back-pressure congestion control based on local information", in IEEE Transactions on Automatic Control, Vol. 40, 1995.

[45] A.Rao, S.Ratnasamy, C.Papadimitriou, S.Shenker, and I.Stoica, "Geographic routing without location information", in The Proceeding of ACM Mobilcom'03, 2003.

[46] S.Chen, G.Fan, and J.Cui, "Avoid void in geographic routing for data aggregation in sensor networks", in International Journal of Ad Hoc and Ubiquitous Computing(IJAHUC), Special Issue on Wireless Sensor Networks, 2006.

[47] B.Karp and H.Kung, "GPSR: greedy perimeter stateless routing for wireles networks", in The Proceeding of ACM Mobilcom'00, 2000. 